\title{
Derivative recovery and a posteriori error estimate for extended finite elements
}

\author{
Stéphane Bordas ${ }^{* \dagger}$ Marc Duflot ${ }^{\ddagger}$
}

November 6, 2006

\begin{abstract}
The goal of this work is to devise a simple and effective local a posteriori error estimate for partition of unity enriched finite element methods such as the extended finite element method (XFEM). In each element, the local estimator is the $L_{2}$ norm of the difference between the raw XFEM strain field and an enhanced strain field computed by extended moving least squares (XMLS) derivative recovery obtained from the raw nodal XFEM displacements. The XMLS construction is taylored to the nature of the solution. The technique is applied to linear elastic fracture mechanics, in which near-tip asymptotic functions are added to the MLS basis. The XMLS shape functions are constructed from weight functions following the diffraction criterion to represent the discontinuity. The result is a very smooth enhanced strain solution including the singularity at the crack tip. Results are shown for two and three dimensional linear elastic fracture mechanics problems in mode I and mixed mode. The effectivity index of the estimator is always close to 1 . and improves upon mesh refinement. It is also shown that for the linear elastic fracture mechanics problems treated, the proposed estimator outperforms one of the superconvergent patch recovery technique of Zienkiewicz and Zhu, which is only $C_{0}$. Parametric studies of the general performance of the estimator are also carried out.
\end{abstract}

\section{Introduction}

Determining the accuracy of a numerical solution is essential, both to gain a better understanding of the numerical method used, and to measure its reliability in the context of real-world problems. This paper presents an error estimator for partition of unity enriched finite element methods such as the extended finite element method (XFEM) based on derivative recovery with intrinsically enriched eXtended Moving Least Squares (XMLS) approximants and diffraction. The effectivity of the proposed estimator on two and three-dimensional linear elastic fracture mechanics problems treated with an extended finite element formulation is shown through 2D and 3D numerical examples. MLS derivative recovery in finite elements was first proposed in Reference 44 of which this work is a generalization.

The extended finite element method (XFEM) is among partition of unity methods [27, 7, and has been extensively put to work for problems with discontinuities, since 1999 10. Typically, XFEM and related methods add, locally, special functions to the FE approximation, in order to better approximate particular features of a problem. For problems with discontinuities in the primal dependent variable (cracks [29 28] 13], fluid-structure interaction [26]), discontinuous functions are added. To handle discontinuities in the dual unknown (multi-materials [41, solidification [16], biofilms [35], multi-phase flow [15], Stokes flow [47]), functions with discontinuous derivatives enhance the standard basis. These additional functions allow to model the discontinuous features without recourse to a conforming mesh, while retaining an exact representation of the discontinuities, to which the mesh need not conform. In addition to discontinuities, special functions can be added to tell the approximation about a known chacacteristic of the exptected solution, around crack tips or boundary layers for instance. This increases accuracy for coarse

*University of Glasgow, Civil Engineering, Rankine building, G12 8LT email: stephane.bordas@alumni.northwestern.edu Tel. +44 (0) 141330 5204, Fax. +44 (0) 1413304557 http://www.civil.gla.ac.uk/ bordas

† corresponding author

‡CENAERO, Avenue Jean Mermoz 30 B-6041 Gosselies, Belgium marc.duflot@cenaero.be 
meshes, and improves the convergence rate. The extended finite element was coupled with the level set method, and the latest results are given in Reference [17. Hansbo and Hansbo came up with yet another technique to describe strong discontinuities within finite elements [22] whose kinematics was proved equivalent to that of XFEM [5].

Three types of procedures are currently available for deriving error estimators. They are either residual based, recovery based, or constitutive behaviour based. The residual based error estimators were first introduced by Babuška and Rheinboldt in 1978 [6] and have been since used very effectively and further developed by many others. Ainsworth and Oden made substantial progress with the introduction of so called residual equilibration in 1992-1993 [3, 1]. On the other hand, the recovery based estimates have been first introduced by Zienkiewicz and Zhu in 1987 [49. Again, these were extensively improved by the introduction of new recovery processes. These authors have introduced the so called Superconvergent Patch Recovery (SPR) in 1992 [50 51. This method has produced a very significant improvement of performance of recovery based methods. Finally, the French team of Professor Ladevèze devised the error on constitutive behaviour [24]. The book [4] is an excellent reference on error estimates, as is the review paper [2].

Although enriched finite element methods have been very successful in solving problems with singularities and discontinuities, to the authors' knowledge, the only work on a posteriori error estimates for such methods is by the group of Professor Strouboulis in the context of the generalized finite element method (GFEM) 37 38, 39 48.

In general, the exact solution to a problem is unknown. In order to approximate the approximation error of a numerical technique, an enhanced solution is required. How should one go about constructing improved (recovered) fields from an XFEM solution? The use of SPR techniques would be possible, provided the existence, and location of superconvergent points $[8$ was known. Additionally, such recovery techniques perform poorly in regions of high gradients, which does not make it attractive to solve fracture problems. The next possibility that comes to mind is the use of smoothing techniques. One of the simplest and versatile fitting method, is the moving least squares approximation. A major point in favor of using MLS approximants is their ability to include special functions such as jumps, kinks, and any other analytic function such as those commonly used in XFE approximations. We call this enhanced recovery extended moving least squares recovery (XMLS recovery).

The method proposed in this paper is rooted in recovery based methods and extends the work of Reference [4] to partiton-of-unity-enriched finite elements. We compute the recovered (smoothed) field and its spatial derivatives using the nodal values of the primary dependent variable to construct local, enriched, MLS approximations. In a given element, the error estimate is the $L_{2}$ norm of the difference between the raw (XFEM) strain field and an enhanced strain field. The enhanced strain field is computed using the raw nodal XFEM displacements through extended (enriched) MLS derivative recovery (XMLS), where the weight function is computed through the diffraction criterion, and the basis incorporates near-tip fields spanning the linear elastic fracture mechanics solution space. This method is general, because it does not require the existence of superconvergent points, can handle arbitrary enrichment schemes through intrinsic enrichment of the MLS basis and leads to very smooth recovered derivatives thanks to the MLS approximation. It is simple, since the smoothed displacement (strain) field is constructed locally by a simple MLS approximation. It is accurate and the efficiency index of the obtained estimator is close to unity, as numerical studies show. Additionally, it outperforms the superconvergent patch recovery technique of Reference [4] for the fracture mechanics examples studied, which is no surprise since the latter estimator yields only a $C_{0}$ smoothing of the strain. One drawback of the approach is its computational cost, since the enriched MLS basis comprising seven terms must be employed everywhere in the domain. This could be remedied by only enriching around the crack tip, and transitioning to a standard approximation far from the crack.

An outline of the remainder of this paper is as follows. The basics of the extended finite element method and partition of unity methods recalled in Section 2 In Section 3 the construction of moving least squares approximants on a cloud of points is shown, the derivative recovery is explained, and error measures defined. Section 4 solves two-dimensional fracture mechanics problems with the extended finite element method, and computes the associated approximation error distribution through extended moving least squares recovery. First, a mode I problem is studied. Structured meshes are first considered, for which the influence of the XFEM and MLS enrichment radii are studied. The effectivity of the proposed error estimator is also compared to one of the strandard superconvergent patch recovery technique of Zienkiewicz and Zhu. Then, the performance of the estimator is studied on unstructured meshes. A mixed mode crack problem is studied next, for both structured and unstructured discretizations. Section 5] studies the performance of our method on three-dimensional crack problems in mode I and mixed mode. Finally, in Section [6] conclusions are drawn from the work, and extensions proposed. 


\section{Extended finite element method}

The extended finite element method introduced by Belytschko and Black 10] and improved in Reference [29] relies on partition of unity enrichment 7 [27. Other partition of unity enriched finite element methods are the generalized finite element method (GFEM) 37 [38 and Nitsche's method [22], although the latter's kinematics is identical to that of the XFEM [5]. The key idea is to locally add arbitrary special functions to the finite element basis. These functions can make the approximated field or its derivative discontinuous inside an element, thereby releasing the requirement for the mesh to conform to discontinuities and making the method quite suitable to treat fracture mechanics problems. Additionally, the functions added can help capture a known local character in the sought solution. For instance, near-tip crack tip fields of linear elastic fracture mechanics can be added to the approximation basis. This gives the method the ability to achieve good accuracy with relatively coarse meshes. Note that partition of unity has also been applied in a so-called meshfree context, through extrinsic enrichment [19] 46], intrinsic enrichment [43] or weight function enrichment [18. One of the most recent examples for fracture of quasi-brittle materials using meshfree methods with extrinsic enrichment is shown here: 33].

Much work has been done since the seminal work of Professor Belytschko's team on the XFEM. Particularly, the notion of geometrica 1 enrichment was introduced [23, 9], in which the enriched region becomes of fixed measure upon mesh refinement, as opposed to shrinking to zero as was so in the original version of the method. This novelty confers the modified XFEM with optimal convergence rates for linear elastic fracture mechanics. This geometric enrichment method will be used consistently in this paper, except otherwise stated.

Define $\mathcal{N}$ as the set of all nodes in the domain. Nodes whose support is bisected by a crack form set $\mathcal{N}_{c r}$. For topological enrichment (Figure 1), nodes whose support contains the tips are grouped in set $\mathcal{N}_{\text {tip }}$. For geometric enrichment (Figure 21), set $\mathcal{N}_{t i p}$ contains all nodes in a disk centered on the crack tip, with a radius fixed in advance, but problem dependent.

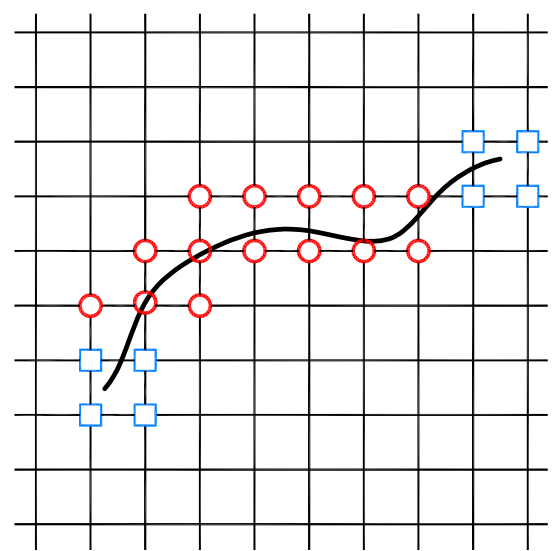

(a) structured mesh of quadrangles

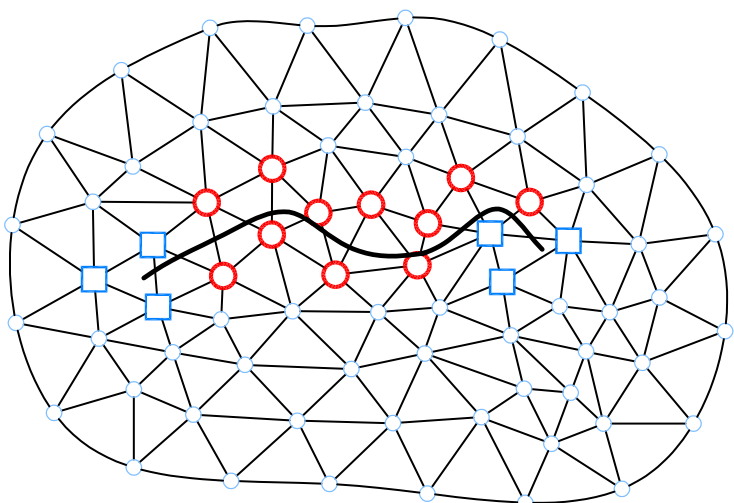

(b) unstructured mesh of triangles

Figure 1: Selection of enriched nodes. Circled nodes ( $\operatorname{set} \mathcal{N}_{c r}$ ) are enriched by the step function whereas the squared nodes (set $\mathcal{N}_{\text {tip }}$ ) are enriched by the crack tip functions.

The finite element shape functions are noted $N_{I}(\mathbf{x})$ and $\widetilde{N}_{J}(\mathbf{x})$ and $\mathbf{u}_{\mathrm{I}}, \mathbf{a}_{\mathrm{J}}$ and $\mathbf{b}_{\alpha \mathrm{K}}$ are the displacement and enrichment nodal variables, respectively. The partition of unity associated with the enrichment, $\widetilde{N}_{J}(\mathbf{x})$ may differ from the $N_{I}(\mathbf{x})$ used for the standard part of the displacement approximation 2 . In this paper, both sets of shape functions are identical and are the standard shape functions of the underlying finite element approximation: threenoded triangles in the 2D examples, four-noded tetrahedra in the 3D examples.

\footnotetext{
${ }^{1}$ as opposed to topological

${ }^{2}$ for instance, quadratic shape functions $N_{I}(\mathbf{x})$ but linear shape functions $\widetilde{N}_{J}(\mathbf{x})$ were used for six-noded triangular elements 36 .
} 
Let $H(\mathbf{x})$ be the modified Heaviside function which takes the value +1 above the crack and -1 below the crack. Finally, note $B_{\alpha}(\mathbf{x})$ a basis that spans the near tip asymptotic fields:

$$
\mathbf{B} \equiv\left[B_{1}, B_{2}, B_{3}, B_{4}\right]=\left[\sqrt{r} \sin \frac{\theta}{2}, \sqrt{r} \cos \frac{\theta}{2}, \sqrt{r} \sin \frac{\theta}{2} \cos \theta, \sqrt{r} \cos \frac{\theta}{2} \cos \theta\right]
$$

With these definitions, the XFEM approximation for a crack in linear elastic fracture mechanics reads

$$
\begin{aligned}
\mathbf{u}^{h}(\mathbf{x})= & \sum_{I \in \mathcal{N}} N_{I}(\mathbf{x}) \mathbf{u}_{I}+\sum_{J \in \mathcal{N}_{c r}} \tilde{N}_{J}(\mathbf{x})\left(H(\mathbf{x})-H\left(\mathbf{x}_{J}\right)\right) \mathbf{a}_{J} \\
& +\sum_{K \in \mathcal{N}_{t i p}} \tilde{N}_{K}(\mathbf{x}) \sum_{\alpha=1}^{4}\left(B_{\alpha}(\mathbf{x})-B_{\alpha}\left(\mathbf{x}_{K}\right)\right) \mathbf{b}_{\alpha K}
\end{aligned}
$$

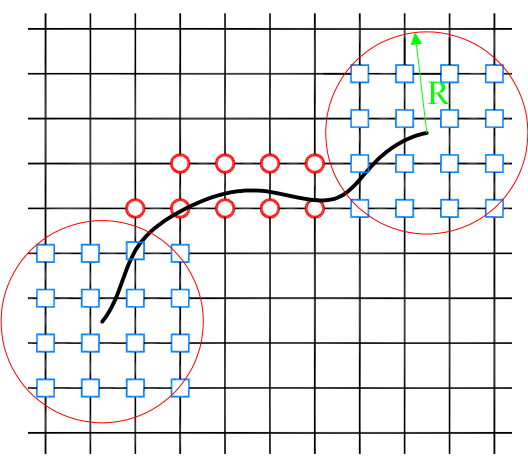

(a)

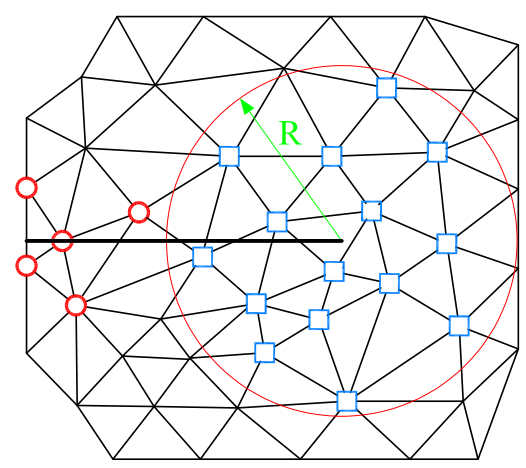

(b)

Figure 2: Another scheme for selection of enriched nodes for $2 \mathrm{D}$ crack problem. Circled nodes (set of nodes $\mathcal{N}_{c r}$ ) are enriched by the step function whereas the squared nodes (set of nodes $\mathcal{N}_{\text {tip }}$ ) are enriched by the crack tip functions. The enrichment radius $r_{e n r}^{X F M}$ is here equal to $R$.

From this enriched approximation (2), the Bubnov-Galerkin procedure yields the standard discrete system of equations $\mathbf{K d}=\mathbf{f}$. The numerical integration for elements cut by the discontinuity or containing the crack tip requires subdivision, except for very special cases 45]. Interested readers can refer to [32] 14] among other sources for details on the extended finite element method 3

\section{Diffracted extended moving least squares recovery}

\subsection{Moving Least Squares approximations}

Moving Least Square approximations (MLS) were originally created by mathematicians to fit and construct surfaces 25. This fitting method has been used to construct shape functions for meshfree methods [12. As introduced above, the idea is to use the raw XFEM displacements to compute an enhanced strain field. The enhanced strain field is taken as the derivative of the MLS smoothing of the raw XFEM displacements. The latter shall be indicated with a superscript $h$, the enhanced by a superscript $s$ (for smooth).

Let us denote by $\mathcal{N}_{X M L S}$ the set of points composed by the XFEM nodes and the points on either sides of all intersections of the crack with the XFEM mesh. These additional points are shown in Figure 3 Note that in [4, the authors use only the FEM nodes as MLS support points; in their method, the mesh then conforms with the crack faces.

\footnotetext{
3 the documents are accessible online at http://www.civil.gla.ac.uk/ bordas
} 
Discontinuous enriched

- tip enriched

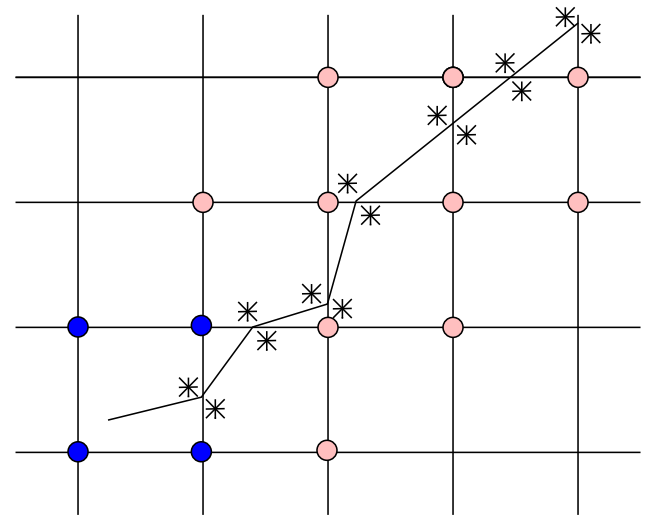

Extra points added on either side

* of the crack, at the intersection

points with the mesh

Figure 3: Points added at all intersections of the crack with the XFEM mesh. The union of these points with the XFEM nodes forms set $\mathcal{N}_{X M L S}$, containing all the nodes used to support the XMLS approximation. Adding nodes along the crack improves its representation by the diffraction method and decreases the size of the domains of influence (smoothing length) required for analysis. 
The XFEM displacement approximation writes as in Equation (2) with $\widetilde{N}=N$. At a node $\mathbf{x}_{I}$, we note the nodal XFEM displacement vector $\mathbf{u}_{I}^{h}=\mathbf{u}^{h}\left(\mathbf{x}_{I}\right)$. The strain field is deduced by the strain-displacement relations. We define the symmetric gradient partial differential operator $\mathcal{D}(\cdot)$

$$
\varepsilon^{h}=\frac{1}{2}\left(\nabla+\nabla^{T}\right) \otimes \mathbf{u}^{h}=\mathcal{D} \otimes \mathbf{u}^{h}
$$

In the following, quantities in square brackets $[\cdot]$ and curly brackets $\{\cdot\}$ are matrix operators and vectors of nodal values, respectively. Let $\mathbf{x}$ be an arbitrary point in $\Omega$, point $\mathbf{x}$ belongs to the domain of influence of $n_{\mathbf{x}}$ points of set $\mathcal{N}_{X M L S}$ defined in figure 3 With these notations, the smoothed displacement and strain fields write

$$
\begin{aligned}
& \mathbf{u}^{s}(\mathbf{x})=\quad \sum_{I}^{n_{\mathbf{x}}} \Phi_{I}(\mathbf{x}) u_{I}^{h}=[\mathbf{\Phi}]^{T}(\mathbf{x})\left\{\mathbf{u}^{h}\right\} \\
& \varepsilon^{s}(\mathbf{x})=\sum_{I}^{n_{\mathbf{x}}} \mathcal{D}\left(\Phi_{I}\right)(\mathbf{x}) u_{I}^{h}=[\mathcal{D}][\mathbf{\Phi}]^{T}(\mathbf{x})\left\{\mathbf{u}^{h}\right\}
\end{aligned}
$$

$\Phi_{I}(\mathbf{x})$ is the value of the MLS shape function associated with node $I$ at point $\mathbf{x} .\left\{\mathbf{u}^{h}\right\}$ and $[\boldsymbol{\Phi}]$ are $n_{\mathbf{x}} \times 1$ vectors, $[\mathcal{D}]$ is a $3 \times n_{\mathbf{x}}$ matrix in $2 \mathrm{D}$ and a $6 \times n_{\mathbf{x}}$ matrix in $3 \mathrm{D}$.

The MLS shape functions of the $n_{\boldsymbol{x}}$ points whose domains of influence contain point $\mathbf{x}$ write

$$
\left[\begin{array}{lll}
\Phi_{1}(\mathbf{x}), & \Phi_{2}(\mathbf{x}), \ldots, & \Phi_{n_{\boldsymbol{x}}}(\mathbf{x})
\end{array}\right]=\boldsymbol{\Phi}^{T}(\mathbf{x})=\boldsymbol{p}^{T}(\mathbf{x}) \boldsymbol{A}^{-1}(\mathbf{x}) \boldsymbol{B}(\mathbf{x}) .
$$

- $\mathbf{p}$ is the vector of $m$ basis functions defined in Table 1 Unless otherwise stated, we use the enriched linear basis given in the last row of the table. The approximation writes $\boldsymbol{u}^{h}=\sum_{j=1}^{m} a_{j} \boldsymbol{p}_{j} \equiv \boldsymbol{p}^{T} \boldsymbol{a}$.

- $\boldsymbol{A}$ is an $m \times m$ matrix that needs to be inverted at all points where the MLS functions are required: $\boldsymbol{A}(\mathbf{x})=$ $\sum_{I=1}^{n_{\mathbf{x}}} w_{I}(\mathbf{x}) \boldsymbol{p}\left(\mathbf{x}_{I}\right) \boldsymbol{p}^{T}\left(\mathbf{x}_{I}\right) . \boldsymbol{A}$ is invertible if $n_{\mathbf{x}}>m$ i.e., there are more points whose domains of influence contain $\mathbf{x}$ than basis functions in $\mathbf{p}$, but this is not a sufficient condition.

- $\mathbf{B}$ is an $m \times n_{\mathbf{x}}$ matrix defined by $\boldsymbol{B}(\mathbf{x})=\left[w_{1}(\mathbf{x}) \boldsymbol{p}\left(\mathbf{x}_{1}\right), w_{2}(\mathbf{x}) \boldsymbol{p}\left(\mathbf{x}_{2}\right), \ldots, w_{n_{\boldsymbol{x}}}(\mathbf{x}) \boldsymbol{p}\left(\mathbf{x}_{n_{\boldsymbol{x}}}\right)\right]$

- $w_{I}$ is the weight function associated with point $\mathbf{x}_{I}$. To account for the presence of the discontinuity, the diffraction criterion is used to construct the weight function, as explained below.

For each supporting point $\mathbf{x}_{I}$, a weight function $w_{I}: \Omega \rightarrow \mathbb{R}$ is defined. In this work, the fourth order spline weight function is chosen. In $1 \mathrm{D}$, if $s$ is the normalized distance $(s \geq 0)$ between node $x_{I}$ and point $x$ in the computational domain:

$$
s=\left|\frac{x-x_{I}}{d_{I}}\right|
$$

where $d_{I}$ is the smoothing length of the support domain of node $x_{I}$, also known as domain of influence. The quartic spline function writes

$$
f_{4}(s)= \begin{cases}1-6 s^{2}+8 s^{3}-3 s^{4} & \text { if }|s| \leq 1 \\ 0 & \text { if }|s|>1\end{cases}
$$

In the 2D (3D) examples, we use circular (spherical) domains of influence, such that

$$
w_{I}(\mathbf{x})=f_{4}\left(\frac{\left\|\mathbf{x}-\mathbf{x}_{I}\right\|}{d_{I}}\right)
$$

To account for the presence of the crack, the computation of $s$ is altered following the diffraction method introduced in 11 31] to represent discontinuities in the context of the element free Galerkin method. The basic idea of the diffraction technique is depicted in Figure4 The standard weight function (discontinuities not present) associated with a node evaluated at a point increases when this point becomes closer to the node. When discontinuities exist, the distance calculation is modified to account for them. If a crack hides a point, the weight of a node at this point is diminished. When the line segment [IB] bissected by a crack, the normalized distance of Equation (7) in the definition of the weight function is replaced by the normalized length of the shortest path from I to B passing by a point of the front (path IXB in Figure 4): 


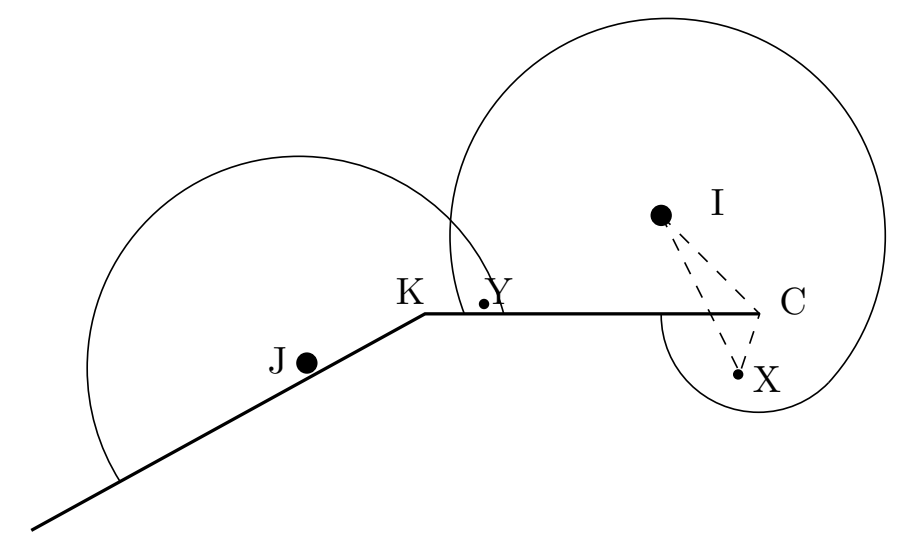

Figure 4: Diffraction method for introducing discontinuities in moving least squares approximations.

Table 1: Some possible basis functions used for MLS approximations. The branch functions $\left(B_{i}\right)_{1 \leq i \leq 4}$ as given by Equation (11).

\begin{tabular}{cccc}
\hline constant & {$[1]$} & {$[1]$} & {$[1]$} \\
\hline linear & {$[1, x]$} & {$[1, x, y]$} & {$[1, x, y, z]$} \\
\hline quadratic & {$\left[1, x, x^{2}\right]$} & {$\left[1, x, y, x^{2}, y^{2}, x y\right]$} & {$\left[1, x, y, z, x^{2}, y^{2}, z^{2}, x y, x z, y z\right]$} \\
\hline linear + tip & - & {$\left[1, x, y,\left[B_{1}(r, \theta), B_{2}(r, \theta), B_{3}(r, \theta), B_{4}(r, \theta)\right]\right]$} & {$\left[1, x, y, z,\left[B_{1}(r, \theta), B_{2}(r, \theta), B_{3}(r, \theta), B_{4}(r, \theta)\right]\right]$} \\
\hline
\end{tabular}

$$
s=\frac{\left\|\mathbf{x}-\mathbf{x}_{c}\right\|+\left\|\mathbf{x}_{c}-\mathbf{x}_{I}\right\|}{d_{I}}
$$

The weight function of node I is then continuous except across the crack. We choose to use additional nodes on each side of the crack 18, as shown in Figure 3] This is by no means mandatory to obtain an accurate representation of the discontinuity, but reduces the support size (smoothing length) required for the calculations, and we make use of this technique in all calculations presented in this paper.

The derivatives of the shape functions may be obtained by differentiating (6). This process involves the evaluation of additional matrices and carrying out several matrix products. This is why the computation of the derivatives of MLS shape functions is a costly operation. The reader is referred to 12 for details on the computation of the successive derivatives of MLS shape functions.

For structured meshes, the smoothing length is fixed in advance to three times the characteristic element size, which ensures that at least seven nodes lie within each domain of influence. This is mandatory for matrix $\mathbf{A}$ to be invertible. For unstructured triangular meshes, the smoothing length is taken to be three times the average characteristic length of the elements sharing the node. For the mode I problem, we also study the influence of the smoothing length.

MLS approximations yield very regular fields on the whole domain. Additionally, the degree of consistency of the approximation is arbitrary ( $p$ refinement, shown in Table 1) and can be increased straightforwardly. More importantly for the object of this paper, special, non-polynomial functions can intrinsically enrich the MLS approximation 
basis, which, similarly to partition of unity enriched methods, makes it possible to closely reproduce a given local behaviour (last row of Table 1). The major difference is that in XFEM, enrichment is local, while in MLS, intrinsic enrichment must be performed on the whole domain, even if the region where it is actually needed is only a small subset of this domain. This increases the computational cost. To avoid this, it is possible to transition from an enriched to a standard MLS approximation, at the cost of additional implementation complexity. An alternative is to enrich the basis extrinsically, leading to partition-of-unity-enriched MLS approximations.

\subsection{Measure of the adequacy of the estimator}

On element $\Omega_{q}$, the approximate error between the raw and the smoothed solution is measured by the norm

$$
e_{\Omega_{q}}^{h s}=\sqrt{\int_{\Omega_{q}}\left\|\varepsilon^{h}(\mathbf{x})-\varepsilon^{s}(\mathbf{x})\right\|^{2} d \mathbf{x}}
$$

The global approximate error is measured by the sum of the element-wise errors on the $n_{\text {elt }}$ elements of the mesh

$$
e^{h s}=\sqrt{\sum_{q=1}^{n_{e l t}} e_{\Omega_{q}}^{h s^{2}}}
$$

Define $e_{\Omega_{q}}^{h e}$ as the following error norm between the raw XFEM solution and the exact solution on element $\Omega_{q}$. We call this error the exact error:

The global exact error is

$$
e_{\Omega_{q}}^{h e}=\sqrt{\int_{\Omega_{q}}\left\|\varepsilon^{h}(\mathbf{x})-\varepsilon^{\text {exact }}(\mathbf{x})\right\|^{2} d \mathbf{x}}
$$

$$
e^{h e}=\sqrt{\sum_{q=1}^{n_{e l t}} e_{\Omega_{q}}^{h e^{2}}}
$$

The global error efficiency index of the estimator is defined as the ratio of the approximate error to the exact error

$$
\theta=\frac{e^{h s}}{e^{h e}}
$$

A good estimator has an efficiency which is close to unity, which means that the measured error is close to the exact error. In other words, the enhanced solution is close to the analytical solution.

\subsection{Recovery algorithm}

The algorithm is particularly simple. The goal is to compute a recovered strain field at each XFEM Gauß point in the mesh. Once the nodal values of the XFEM displacement field are computed at all points in $\mathcal{N}_{X M L S}$ is known, a loop on XFEM Gauß points in the mesh is performed. The XMLS shape functions are calculated based on the locations of the points in $\mathcal{N}_{X M L S}$. For each Gauß point, the values of the XMLS shape functions derivatives are now known. The smoothed strain can be computed using the XFEM displacements of points in $\mathcal{N}_{X M L S}$ and the XMLS shape functions derivatives.

\section{Two-dimensional numerical examples}

\subsection{Introduction}

This section shows the performance of the error estimator for two problems. The domain is a biunit square (bottom left coordinate $[-1,1]$ top right coordinate $[1,1])$. The zone enriched with near-tip fields is a disk centered on the 
crack tip of radius 0.1, unless otherwise noted. A horizontal edge crack of unit length is located along the line $y=0$ and the crack tip is at $(0,0)$. Two loading conditions are assessed (mode I and mixed mode conditions), both for a structured and unstructured mesh of linear triangular elements. For each mesh type, a convergence study is performed, and the effectivity index of the proposed error estimator is analysed, when the analytical solution is known. In particular, the influence of the enrichment radius on the effectivity index is evaluated, and, for fixed enrichment radius, it is verified that the effectivity is close to one.

For each test case, six figures are shown, labeled (a) through (f).

(a) Mesh used for the XFEM calculation

(b) Raw deformation field computed with XFEM: $\varepsilon^{h}$

(c) Displacements of the nodes in $\mathcal{N}_{X M L S}$ obtained by XFEM: $\mathbf{u}_{I}^{h}=\mathbf{u}_{h}\left(\mathbf{x}_{I}\right)$

(d) Smoothed deformation obtained by the recovery algorithm: $\varepsilon^{s}$

(e) Values $\epsilon_{y y}(x=0)$ for both the raw XFEM deformation field and the smoothed deformation field obtained through MLS derivative recovery. The exact strain field is also plotted, when available.

(f) The distribution of the error norm $e_{\Omega_{q}}^{h s}$ of Equation (11) in the domain. In a remeshing algorithm, in each element, this error norm would be compared to a limit error, and would determine whether an element should be enlarged, or shrunk.

\subsection{Edge crack under mode I loading}

This section concerns the case of the edge crack in mode I loading subjected to uniform tension applied at infinity and described in Section 4.1 Traction boundary conditions representing the exact stress state due to the far field are applied on the boundary, while the bottom left corner is restrained in both directions and the bottom right corner in the vertical $(y)$ direction. The MLS basis can represent exactly the exact solution to the problem.

\subsubsection{Structured mesh}

First, the problem is solved on a moderately fine, regular structured mesh. Two cases are compared to show the necessity for the addition of the near-tip fields to the MLS basis. In the first, the four additional functions associated with the asymptotic fields are added to the MLS basis upon computation of the recovered derivative. In the second, only a linear MLS basis is used.

With near-tip fields in the MLS basis The results are given in Figure 5 It is clear from Figure 5 (b) and (e) that the raw strain field from XFEM is piecewise continuous while the recovered strain field, computed from the XFEM nodal displacements of Figure 5(c) are smooth, and very close to the exact solution, even on this moderately fine mesh.

Figure [6] shows that the norm of the difference between the raw XFEM strain field and the XMLS recovered strain field converges to zero with a rate close to the optimal rate of 1.0 as the mesh size tends to zero, as long as a fixed area is enriched around the crack tip during mesh refinement. If only the crack tip element is enriched, the convergence rate is 0.46022 , close to $1 / 2$, which is the strength of the crack tip stress singularity. This corroborates the findings of References 23 9]. Five convergence curves are shown, for four values of the XFEM enrichment radius and one for topological enrichment (only the tip element is enriched). Note that the higher the enrichment radius, the lower the error, the straighter the convergence line, and the closer to optimal the convergence rate is, as summarized in Table 3

Figure[7]shows the evolution of the effectivity index with mesh refinement for four values of the XFEM enrichment radius. It is interesting to notice that the effectivity $e$ versus mesh size $h$ curves become closer to the horizontal line $e=1$ with increasing enrichment radius. Additionally, the effectivity of the error indicator clearly becomes closer to unity as the mesh size goes to zero, as is expected. If only the tip element is enriched, the effectivity is not optimal (oscillates about 95\%) and does not converge to 1. with mesh refinement. For coarse meshes, large enrichment radii are necessary to obtain an effective indicator. This is expected, enrichment takes over the standard FEM approximation, which in itself is poorly suited to represent the near-tip behaviour. As the mesh is refined, 


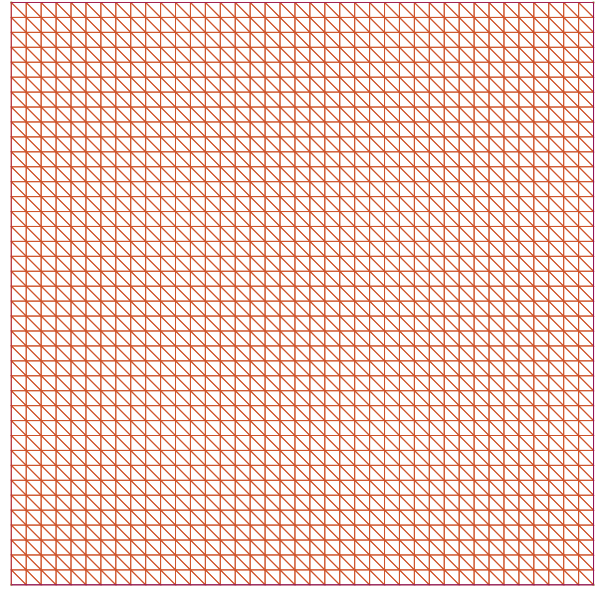

(a) XFEM mesh

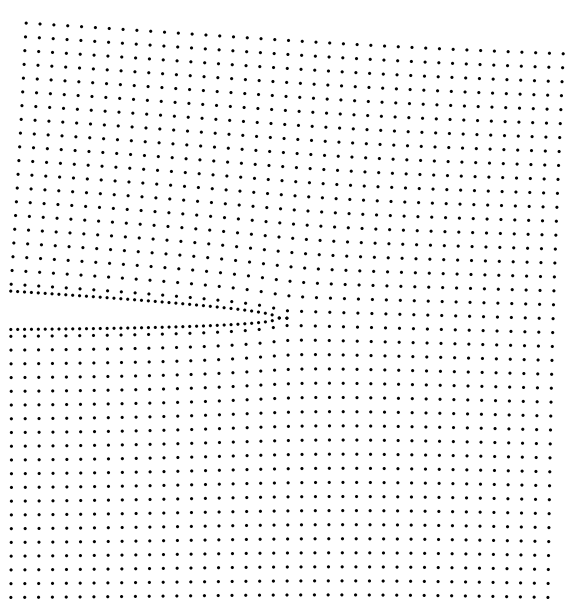

(c) Displacement of the nodes in $\mathcal{N}_{X M L S}$. Only the nodes are represented

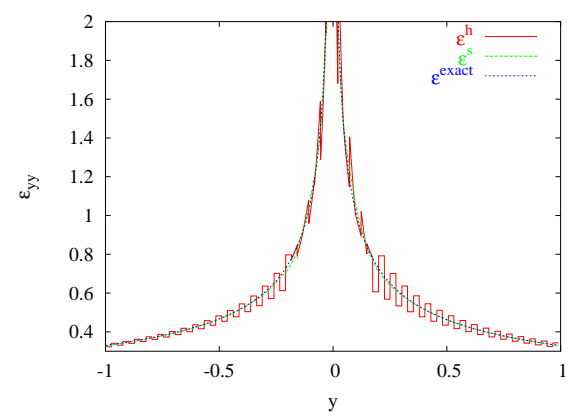

(e) $\epsilon_{y y}(x=0)$

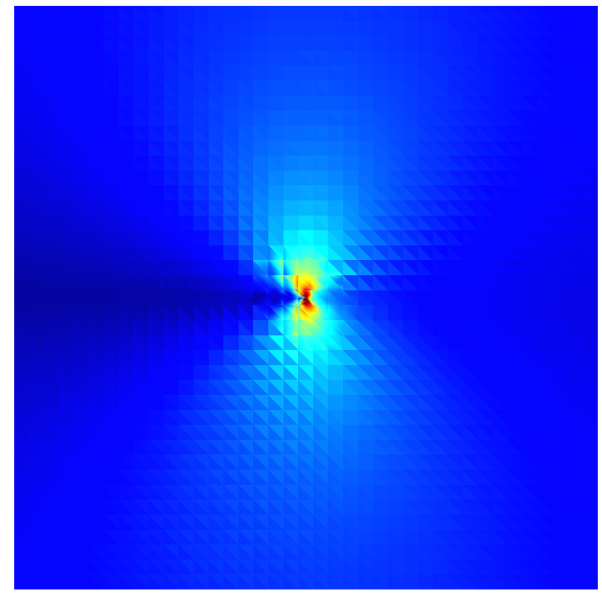

(b) Raw strain field

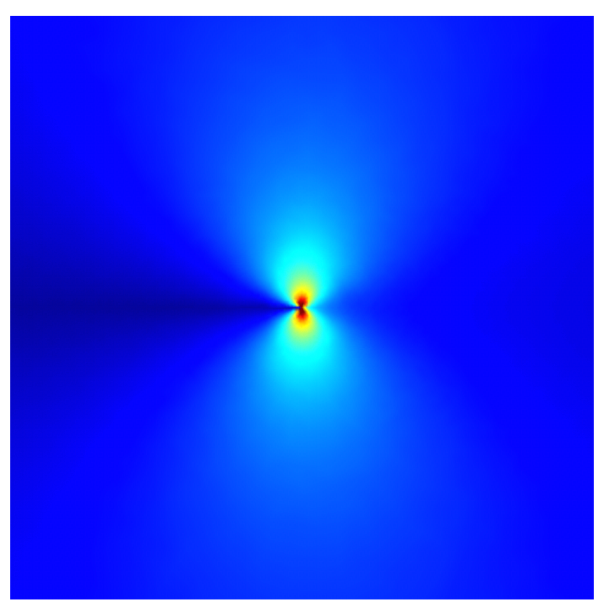

(d) XMLS recovered strain field

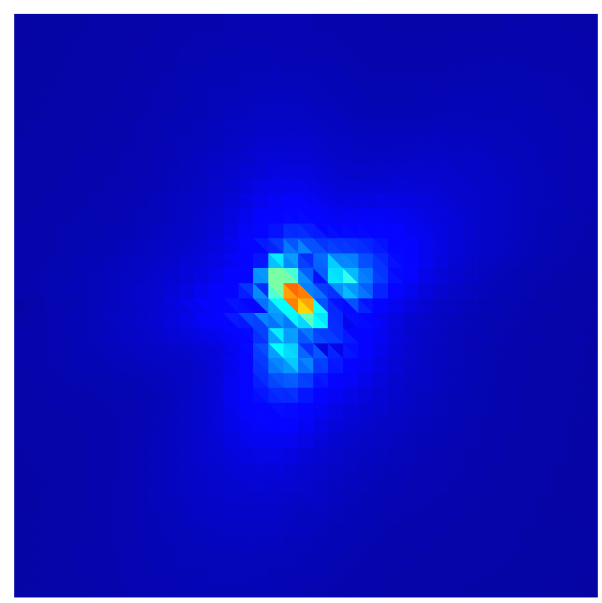

(f) Distribution of $e_{\Omega_{q}}^{h s}$ of Equation (11) on the 1 mesh

Figure 5: Results for a structured, moderately fine meh. 


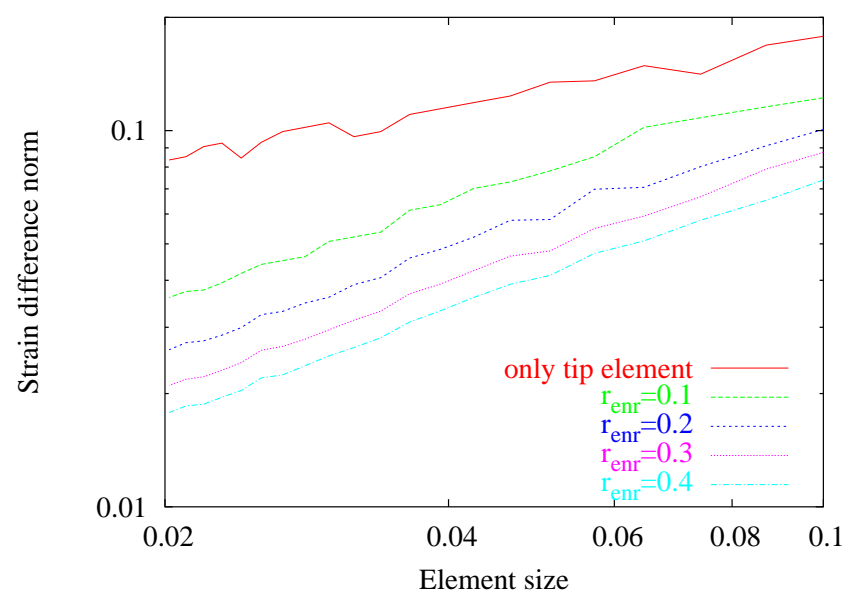

Figure 6: Mode I structured mesh: strain difference convergence with mesh refinement. See Table 3 for the values of the convergence rates. The larger the enrichment radius, the lower the error and the more monotonic the convergence. If only the tip element is enriched (geometrical enrichment), the convergence rate is 0.46022 , for an enrichment radius of 0.4 it reaches 0.91052 , close to optimum.

Table 2: Mode I problem, convergence for the linear and enriched (extended) MLS bases.

\begin{tabular}{cc} 
MLS basis & convergence rate \\
\hline linear & 0.478468 \\
\hline linear + near-tip & 0.81271 \\
\hline
\end{tabular}

the standard FEM part of the approximation becomes more important, and the enriched part less important, which explains the effectivity curves all meeting in the vicinity of 1 . for small $h$ 's.

Without near-tip fields in the MLS basis To motivate the need for the near-tip fields to be included in the MLS basis used for the derivative recovery, the mode I problem is now solved without adding those fields to the MLS basis. When examining Figure 8 and comparing to Figure $[\mathrm{f}$ ), it is clear that the recovered strain field is not as accurate as before. The deformation is more spread out, and the correct strain fringes are not reproduced. Additionally, Figure 9 shows (a) the convergence of the norm of the difference between the raw and the recovered strain field and (b) the convergence of the effectivity index as the mesh size tends to zero. In the figures, both the results for the linear and the full (linear plus near-tip enrichment) MLS basis are presented. It is evident that the convergence rate for the linear basis is asymptotically lower and the error is much higher. Similarly, the error estimate is not effective, since upon mesh refinement, its effectivity diverges from the optimal value of 1 . To conclude, Table 2 compares the convergence rates for the linear and full enrichment bases. Table[3 summarizes the convergence rates obtained for the geometrical and topological XFEM enrichments.

Influence of the MLS enrichment radius In this section, the influence of the MLS radius on the error estimate is investigated. The mode I problem presented in Section 4.2 is revisited with three values of the XMLS 


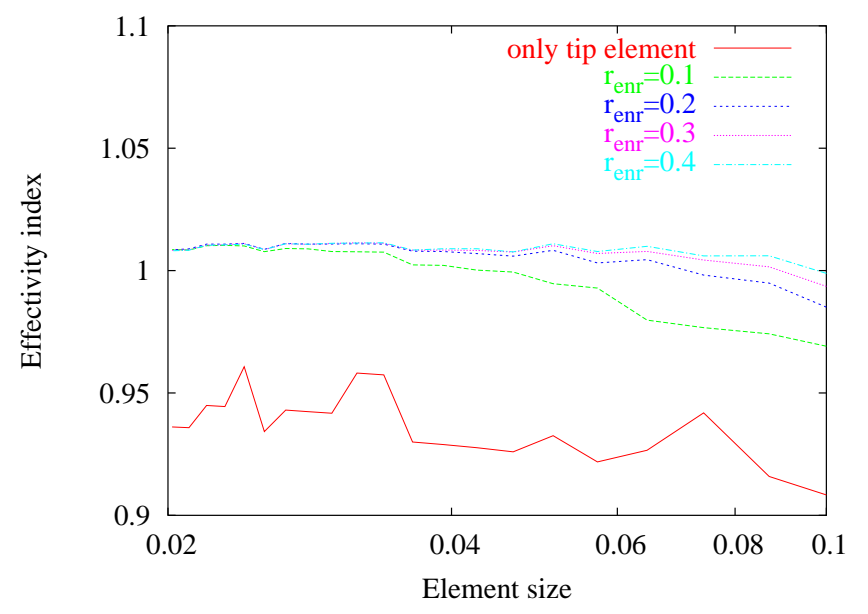

Figure 7: Mode I problem, structured mesh: effectivity index for various XFEM enrichment radii. Enriching only the tip element causes an ineffective error indicator non-convergent to 1. For coarse meshes, higher values of the enrichment radius increases the effectiveness of the indicator. Upon mesh refinement, whatever the enrichment radius, the effectiveness curves meet in the close vicinity of unity.

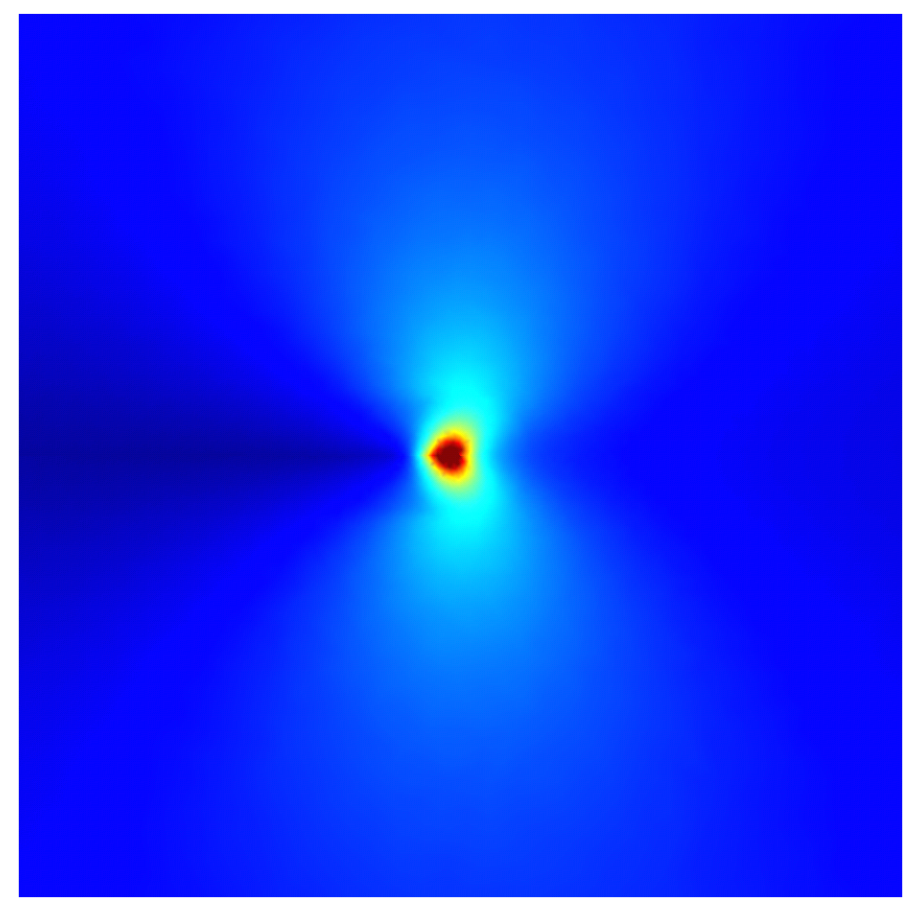

Figure 8: Mode I structured mesh, case of a linear MLS basis used for recovery: smooth (recovered) strain field. 


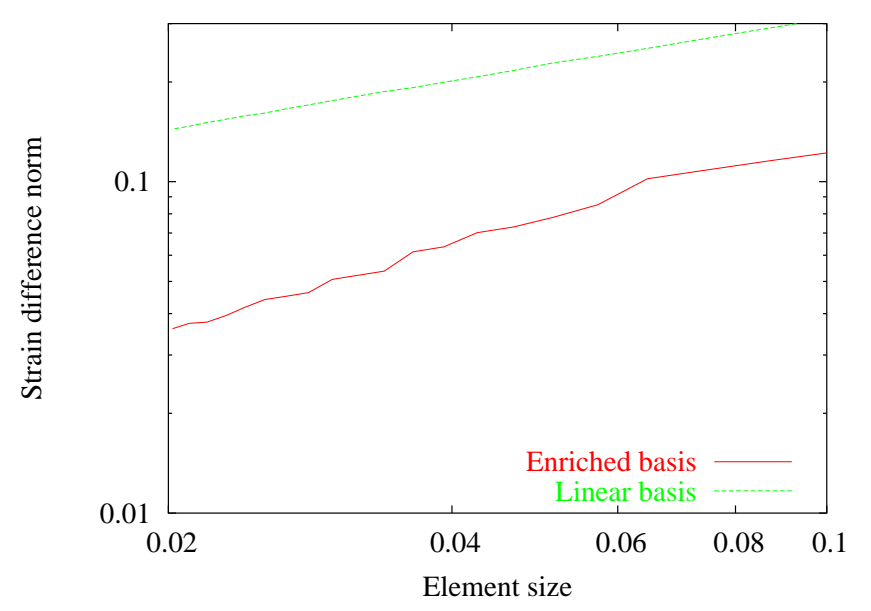

(a) Convergence of the norm of the difference of the raw strain with the recovered strain

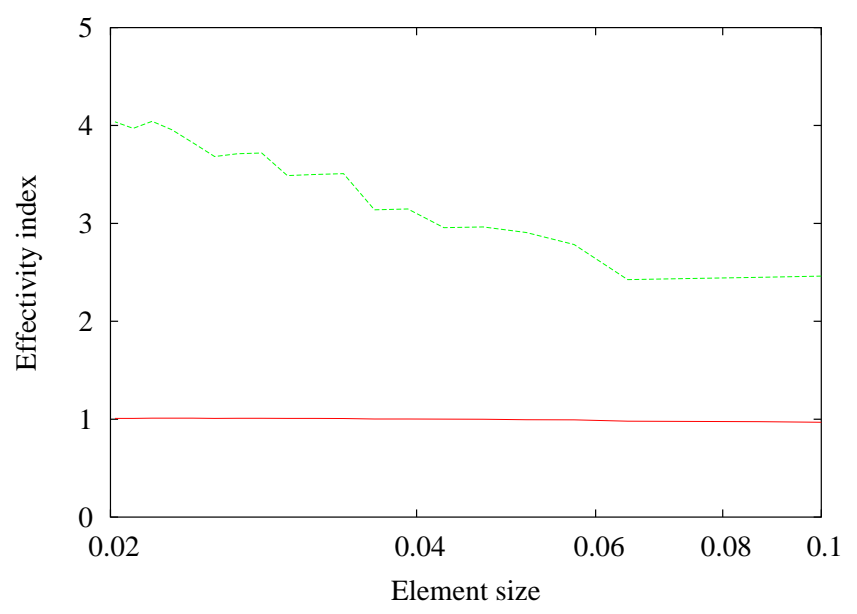

(b) Efficiency index versus mesh size

Figure 9: Mode I problem: linear versus full XMLS recovery basis: convergence of the strain difference and effectivity index with mesh refinement. Notice that the convergence rate is 0.478468 for the linear basis: a value of $1 / 2$ is expected because of the $\sqrt{r}$ singularity. The enriched basis maintains the optimal value of unity, and the overall error is much less than with a linear basis. The defficiency of the linear basis is even more striking when looking at the effectivity index, which does not converge to unity as the mesh size goes to zero.

Table 3: Mode I problem, convergence for various XFEM enrichment radii. See also Figure 6] Close to optimal onvergence is attained for a fixed area enrichment. The results tend to show that the rate of 1 . would be reached if the whole component were enriched.

\begin{tabular}{cc} 
Enrichment radius: $r_{e n r}^{X F M}$ & convergence rate \\
\hline only tip element & 0.46022 \\
\hline 0.1 & 0.81271 \\
\hline 0.2 & 0.86728 \\
\hline 0.3 & 0.90644 \\
\hline 0.4 & 0.91052 \\
\hline
\end{tabular}




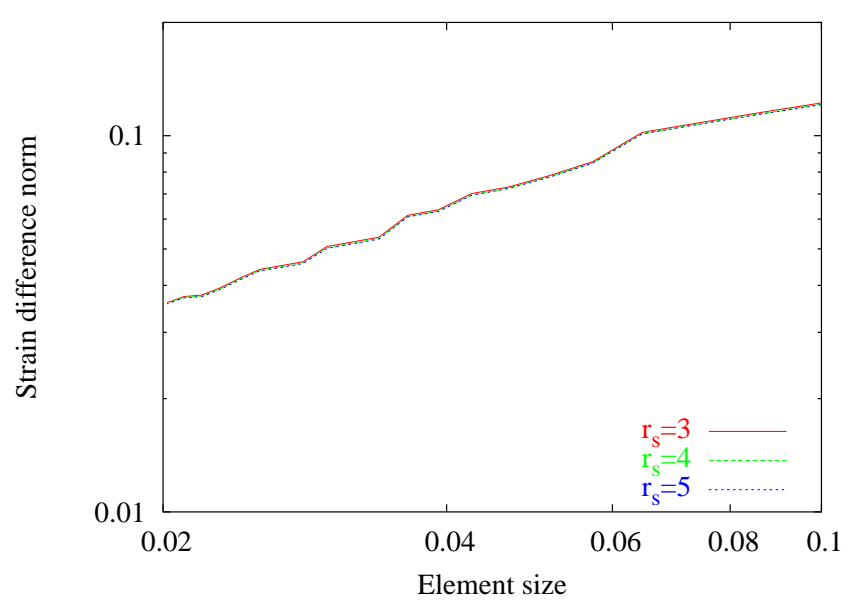

(a) Convergence of the norm of the difference between the raw XFEM

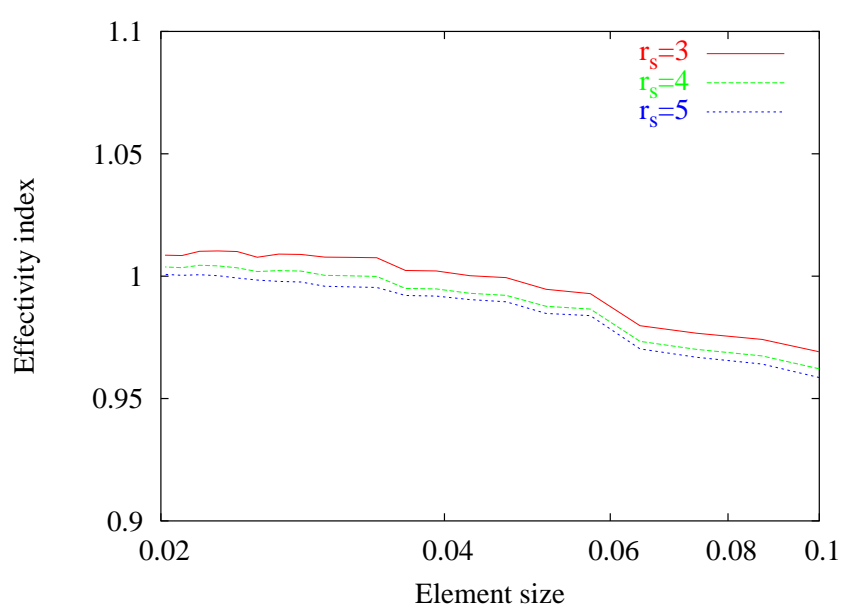

(b) Convergence of the effectivity index with mesh refinement and recovered strain field with mesh refinement

Figure 10: Influence of the MLS radius on convergence of the strain difference and the effectivity index. The convergence curve is unaltered when increasing the XMLS recovery radius, while the effectivity index improves slightly.

recovery radius of influence. If $h$ is the characteristic tip-element, the MLS smoothing length is chosen as $3 h$ (smallest value possible to compute the MLS shape functions, and used previously), $4 h$ and $5 h$. Figure 10 shows that the convergence rate of the norm of the difference between the raw XFEM strain field and the recovered strain field remains the same when increasing the recovery radius, as does the overall value of this error norm. On the contrary, the effectivity index of the error estimate becomes closer to 1. It is the opinion of the authors that this relative increase is too small to justify the increased computational cost incurred when using larger interpolation radii. Table 4 summarizes the convergence rates obtained for various XMLS enrichment radii.

Comparison of the recovered solution with SPR To further motivate the need for extended moving least squares recovery, this section is dedicated to a comparison of the proposed recovered solution with the now standard SPR of Reference [49. Figure 11 compares the smoothed strain results using SPR (a) and XMLS (b) smoothing. The superiority of the XMLS smoothing method is clear for this fracture problem.

\footnotetext{
${ }^{4}$ which we shall name XMLS
}

Table 4: Mode I problem, structured mesh: convergence for various XMLS enrichment radii. $h$ is the characteristic element size around the tip element.

\begin{tabular}{cc} 
MLS smoothing length $r^{X M L S}$ & convergence rate \\
\hline $3 h$ & 0.81271 \\
\hline $4 h$ & 0.81186 \\
\hline $5 h$ & 0.81231 \\
\hline
\end{tabular}




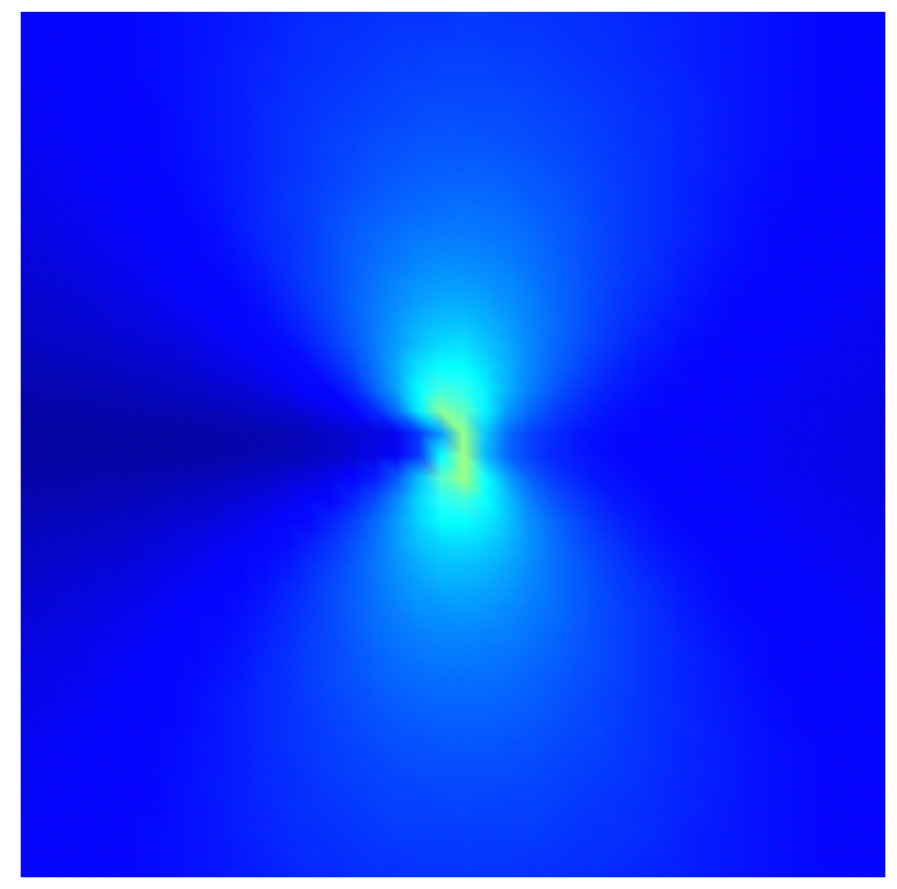

(a) SPR

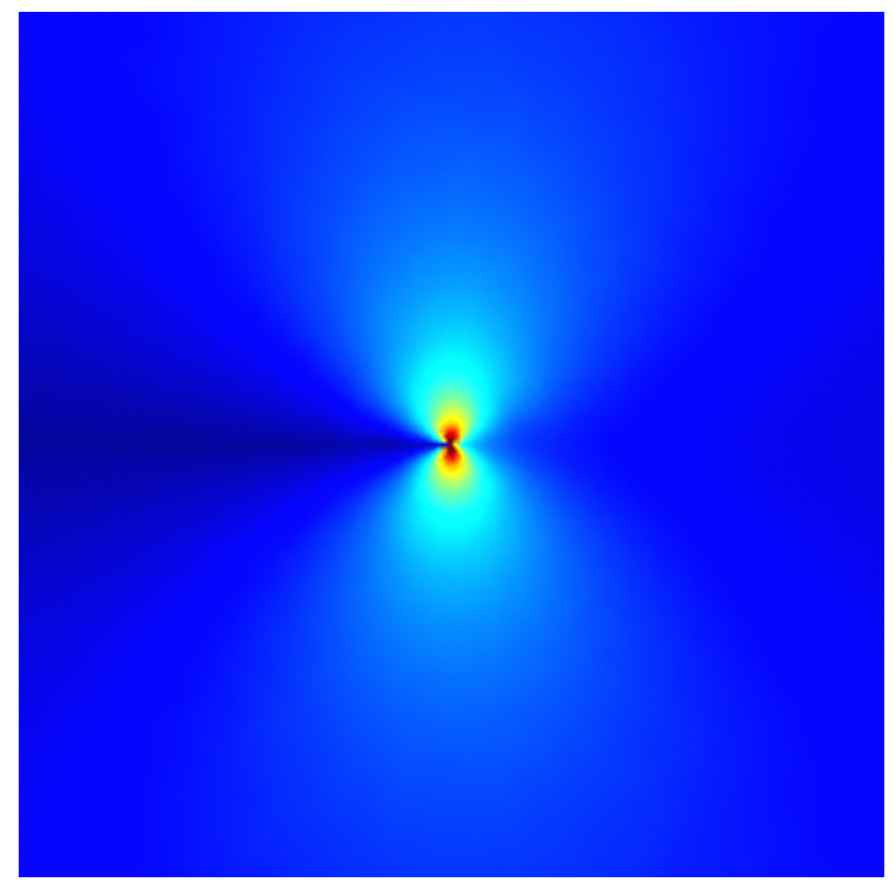

(b) XMLS

Figure 11: Comparison of the superconvergent patch recovery (SPR) error indicator [49] and the proposed extended moving least squares XMLS error indicator. 


\subsubsection{Untructured mesh}

Now that the effectivity of the error indicator was proven on structured mesh, the case of unstructured XFEM meshes is treated. The mesh is generated using the open source software Gmsh 34. Results are shown in Figure 12 Again, the raw strain field obtained from XFEM suffers from the low degree approximation (jumps in Figure 12(e)), where the unstructured nature of the mesh is revealed in the uneven strain steps. On the other hand, the XMLS recovered strain field is very regular, and follows nicely the analytical solution. The smoothness of the recovered field is evident from Figure 12(d). The map shown in Figure 12 (f), showing the difference between the raw and recovered strain field indicates the region where the error indicator advises to refine the mesh.

Now, it is interesting to note that the XFEM results on an unstructured mesh are poorer than their structured mesh counterpart. In particular, the convergence is non monotonic, as shown in Figure 13 Remember that the MLS approximation is non-polynomial (even for a linear basis) and are not exactly integrable using Gauß-LobattoLegendre quadrature. One could wonder if the convergence oscillations depicted in Figure 13 are caused by an inaccurate integration. This is not the case, since the results shown are converged down to $10^{-4}$ precision; additional Gauß points do not substancially decrease the error. The authors speculate that this non-monotonic convergence emanates from the fact that upon unstructured mesh refinement, the position of the tip with respect to the nodes of the tip element varies. It is likely that, for some mesh configuration, the tip falls very close to a node, leading to badly conditioned matrices, and oscillations.

In Figure 14 one can see that the effectivity index is oscillatory, but still remains close to unity as the mesh size goes to zero, as in the structured mesh case. However, we notice more ample oscillations in the effectivity than for the structured mesh. This is certainly due to badly conditionned matrices caused by the tip being close to one of the tip element's nodes as the mesh is unstructurally refined.

\subsection{Edge crack under mixed mode loading}

In this mixed mode loading case, the $x$ and $y$ displacements are fixed on the bottom edge of the square plate, and a horizontal traction $t_{x}=1$ is imposed on the top edge. The MLS basis is unable to represent the solution to this problem exactly, as opposed to the mode I case treated previously.

\subsubsection{Structured mesh}

The mixed-mode problem is first examined using a structured mesh. From Figure 15 several points are to be noticed. Figure 15 b) and (e) show even more clearly than in the mode I case, the irregularity of the raw XFEM strain field. Because of the restraints on the lower edge, the bottom right corner of the domain sees a strain concentration, which leads to a higher error. The error estimate on the whole domain shown in Figure 15(f) shows that the heaviest refinement is to occur in a ball of radius 0.2 centered on the crack tip close to the crack tip. We also note that a mesh adaption step based on this indicator would also advise to refine the mesh at the bottom corners, which is adequate. Since no close form solution is known for this particular mixed-mode problem, Figure 15] (e) only shows the raw and the recovered strain fields, where, again, the smoothness of the recovered field is to be remarked.

It is seen from Figure 16 that the convergence of the $L_{2}$ norm of the strain difference with mesh refinement is quite uniform, with a rate of 0.57994 . Note that the fixture condition at the bottom right corner induces a singularity of strength 0.71 [52] (Chapter 14), a singularity which is not included in the XMLS basis and therefore cannot be expected to be reproduced. The theoretical rate of convergence for this problem is therefore only 0.71 and the value 0.57994 is slightly lower. The error committed here is comparable to that reported in the pure mode I case of the earlier sections.

\subsubsection{Untructured mesh}

The unstructured mesh calculations are shown in Figures 17 and 18 for a moderately fine mesh. The predicted refinement zone is very similar to that of the structured mesh calculations. Studying the convergence of the norm of the difference between the raw XFEM strain and the XMLS recovered strain shows that the optimal rate of unity is almost reached. 


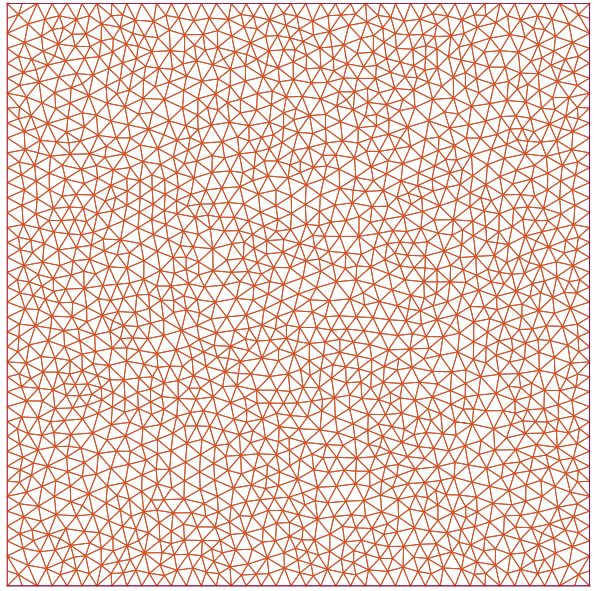

(a) XFEM mesh

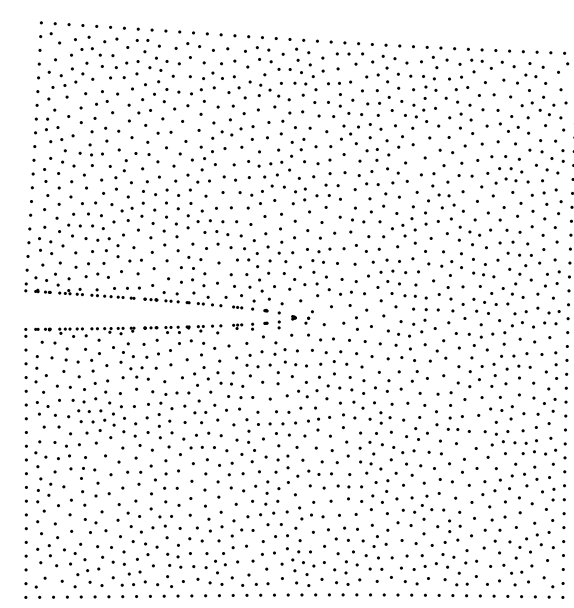

(c) Displacement of the nodes in $\mathcal{N}_{X M L S}$. Only the nodes are represented

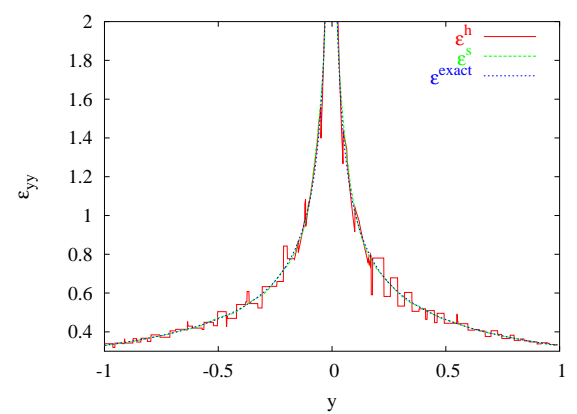

(e) $\epsilon_{y y}(x=0)$

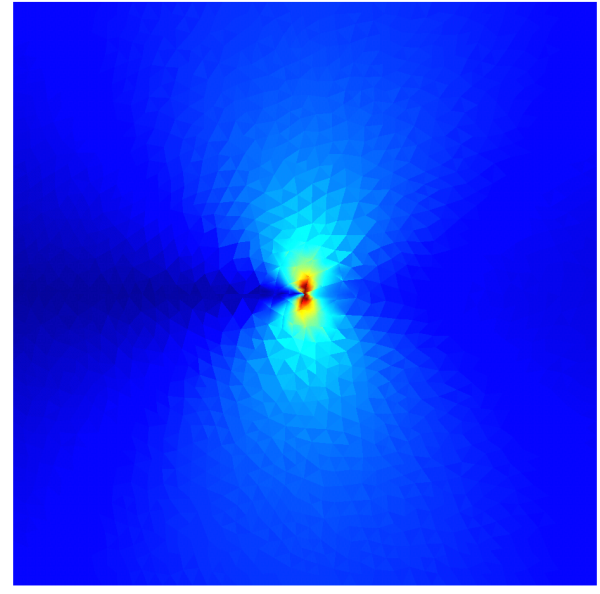

(b) Raw strain field

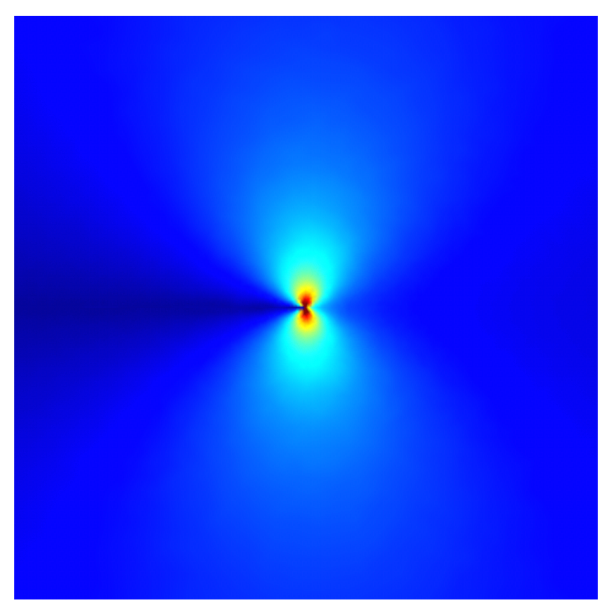

(d) XMLS recovered strain field

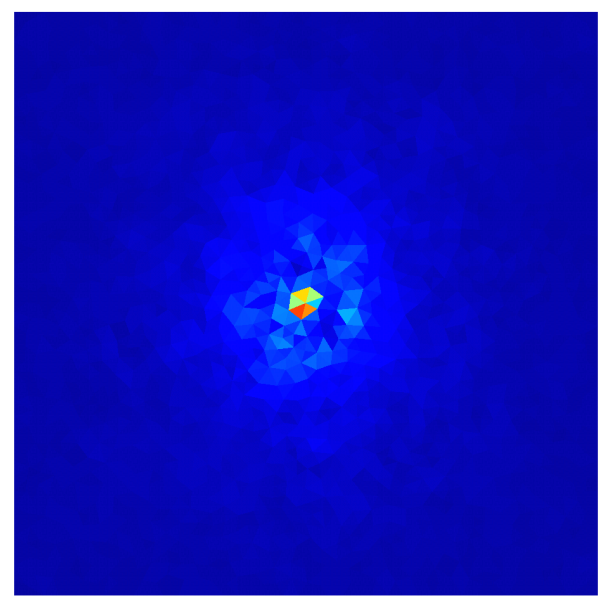

(f) Distribution of $e_{\Omega_{q}}^{h s}$ of Equation 111 on the $17_{\text {mesh }}$

Figure 12: Results for the mode I problem and a moderately fine unstructured mesh 


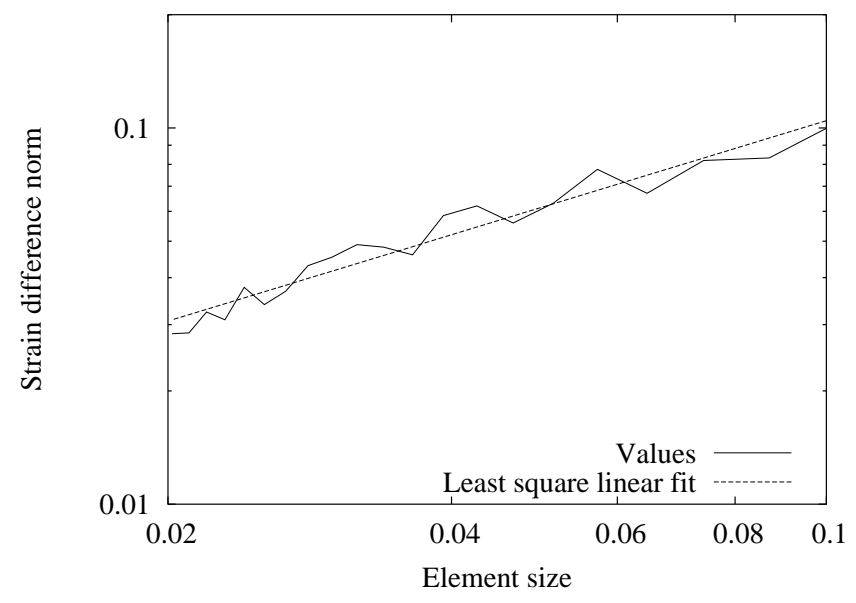

Figure 13: Mode I problem unstructured mesh: strain difference $L_{2}$ norm, convergence slope $=0.76200$, linear triangular elements

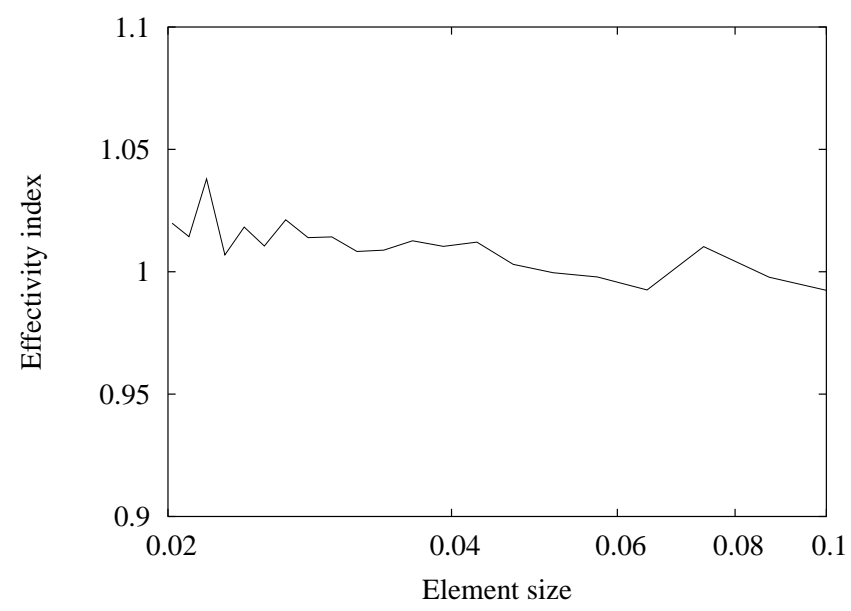

Figure 14: Mode I problem unstructured mesh: effectivity index for linear triangular meshes. 


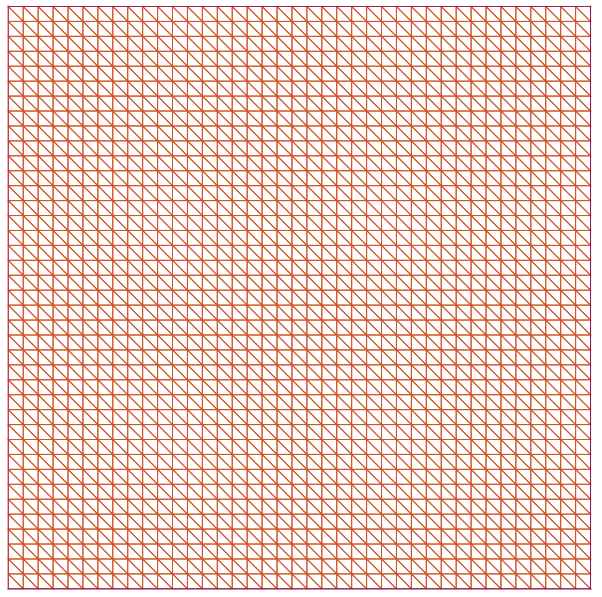

(a) XFEM mesh

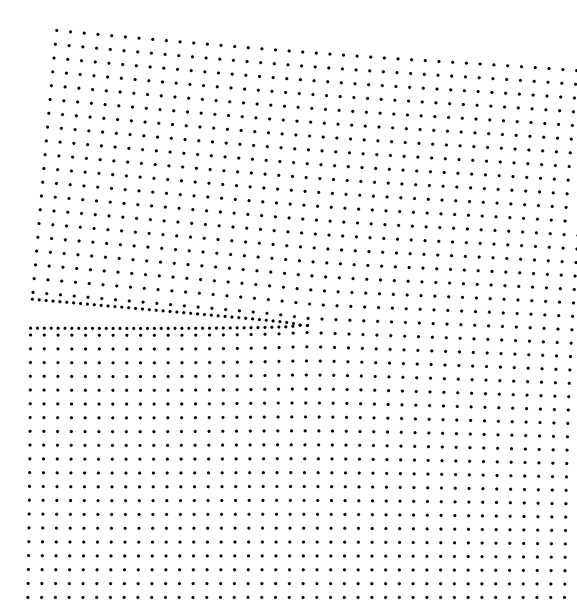

(c) Displacement of the nodes in $\mathcal{N}_{X M L S}$. Only the nodes are represented

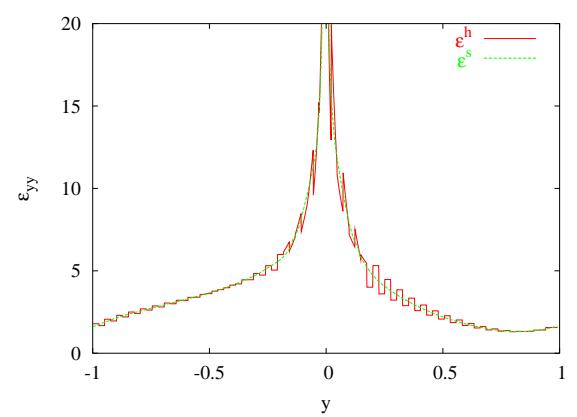

(e) $\epsilon_{y y}(x=0)$

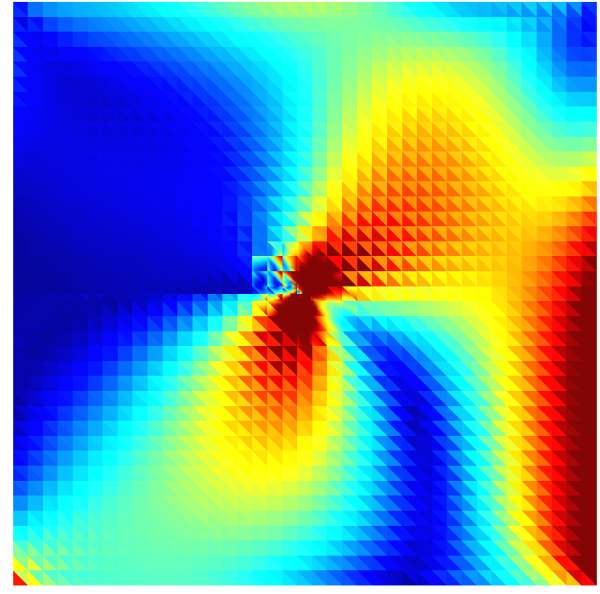

(b) Raw strain field

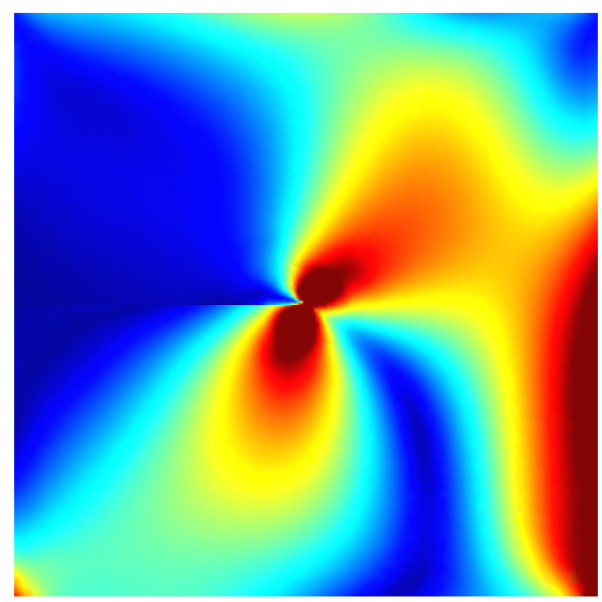

(d) XMLS recovered strain field

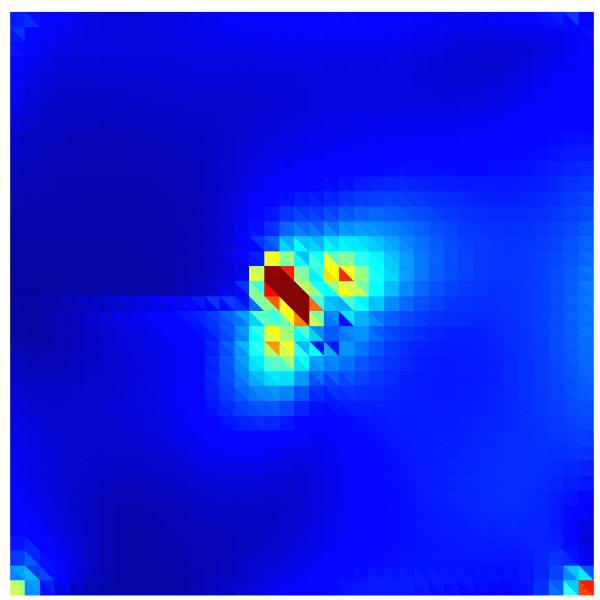

(f) [Distribution of $e_{\Omega_{q}}^{h s}$ of Equation (11) on 19 he mesh

Figure 15: Results for the mixed-mode case and a structured mesh. Notice that because of the restraints on the bottom edge, strain concentration appears at the lower right corner, leading to an increased error prediction in this zone. 


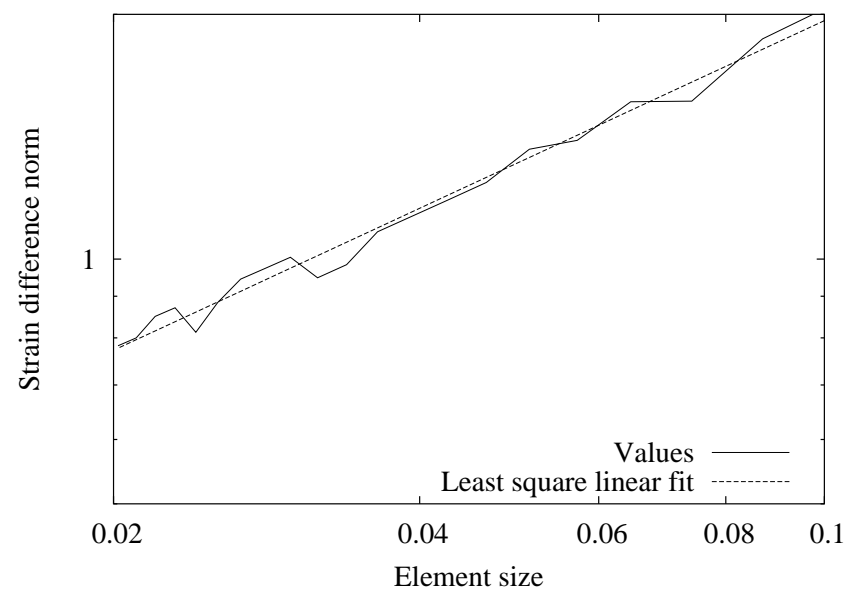

Figure 16: Mixed mode, structured case, strain difference $L_{2}$ norm: slope $=0.57994$

\section{Three-dimensional cracks}

This section presents two three-dimensional crack examples, still in the context of linear elastic fracture mechanics. The domain is $[-1,1] \times[-1,1] \times[-0.5,0.5](x \times y \times z)$. The crack is defined by the equation $y=0, x \leq 0$, its front is along the $z$ axis. We show both the raw and enhanced strain field on the deformed configuration, so that the values on the faces of the crack, and along its front may be better identified.

\subsection{D mode I edge crack}

The geometry is defined as in the introduction to Section 5 and the domain is subjected to uniform uniaxial tension, fixture conditions being added to prevent rigid body modes. Figure 20] compares the deformation field from the XFEM (a) to the smoothed (recovered) field (b) and shows clearly the superiority of the XMLS recovered solution. Figure 19] shows the strain $\varepsilon_{y y}$ along line $x=0$. We believe that using the enhanced strain and stress fields to compute the interaction integrals commonly used to obtain the stress intensity factors, the oscillations in the soobtained SIFs, reported in various papers 30 21, 13, 40, 42] will be reduced. Not only is the enhanced strain field smoothed in the direction normal to the crack front, but also in the tangent direction, which should reduce the amount of oscillations in the SIFs.

\subsection{D edge crack under combined tension and torsion loading}

The geometry is as defined in the introduction of section 5 On face $y=1$, tractions are imposed as follows:

$$
\begin{array}{r}
t_{y}=1 \\
t_{x}=4 z\left(1-x^{2}\right) \\
t_{z}=4 x\left(0.25-z^{2}\right)
\end{array}
$$

On face $y=-1$, tractions are imposed as follows:

$$
\begin{array}{r}
t_{y}=-1 \\
t_{x}=-4 z\left(1-x^{2}\right) \\
t_{z}=-4 x\left(0.25-z^{2}\right)
\end{array}
$$




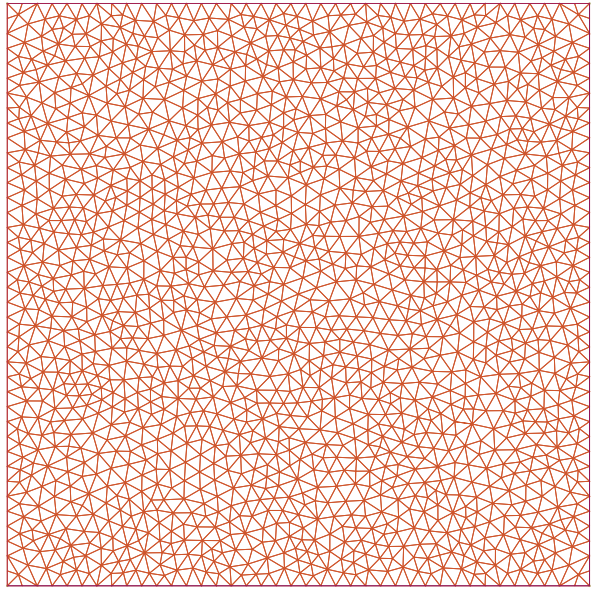

(a) XFEM mesh

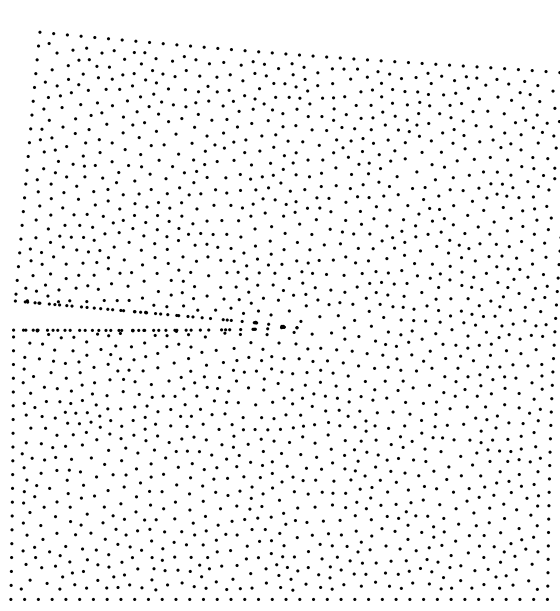

(c) Displacement of the nodes in $\mathcal{N}_{X M L S}$. Only the nodes are represented

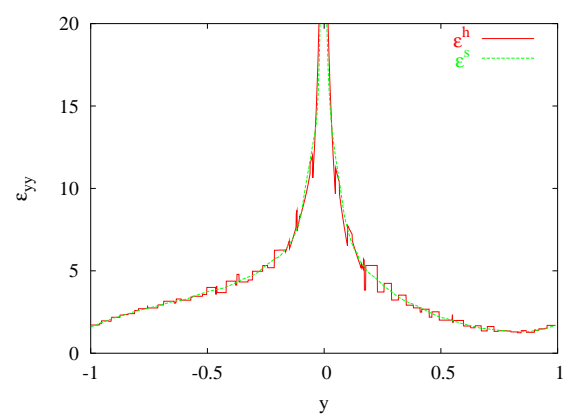

(e) $\epsilon_{y y}(x=0)$

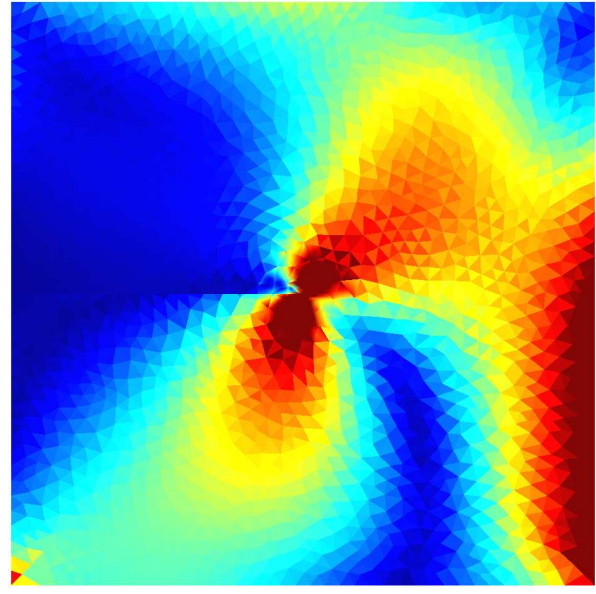

(b) Raw strain field

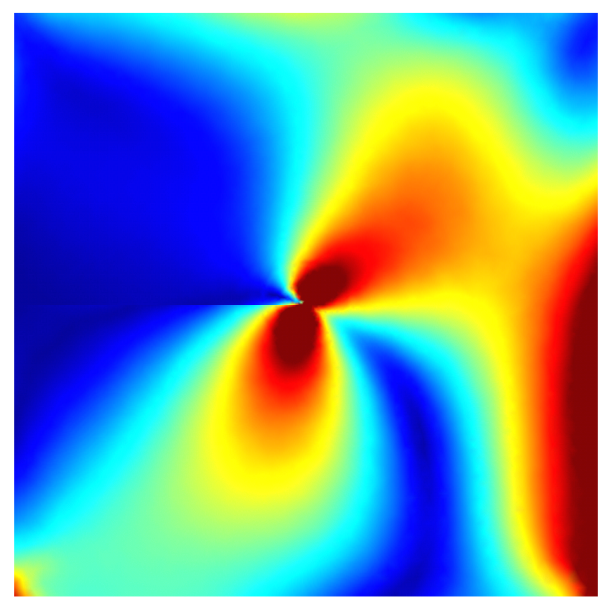

(d) XMLS recovered strain field

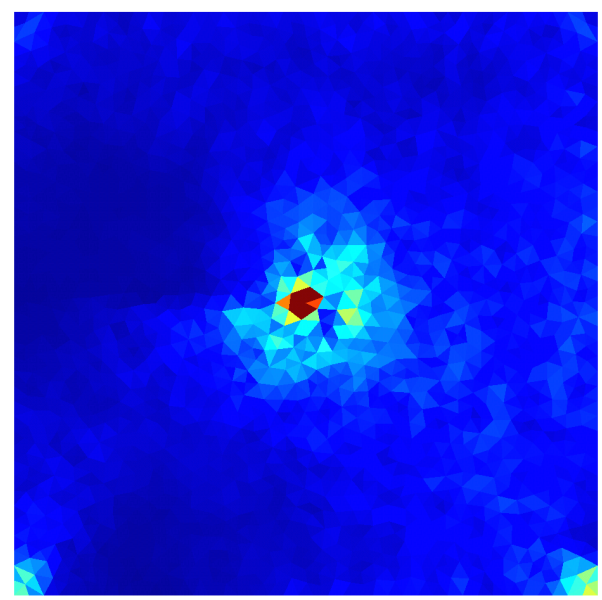

(f) Distribution of $e_{\Omega_{q}}^{h s}$ of Equation (11) on the 21 nesh

Figure 17: Mixed mode problem, unstructured mesh. 


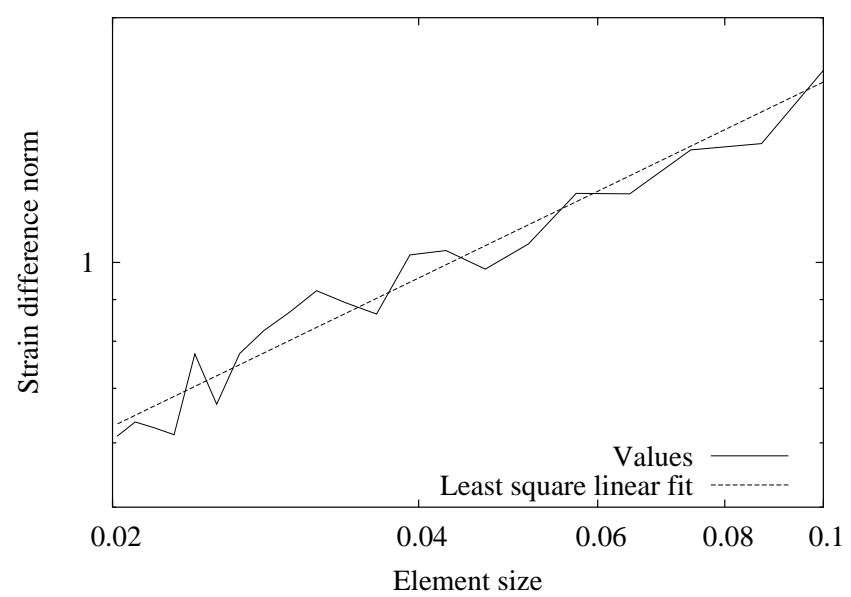

Figure 18: Mixed mode problem, unstructured mesh: strain difference $L_{2}$ norm, convergence at average rate 0.60584

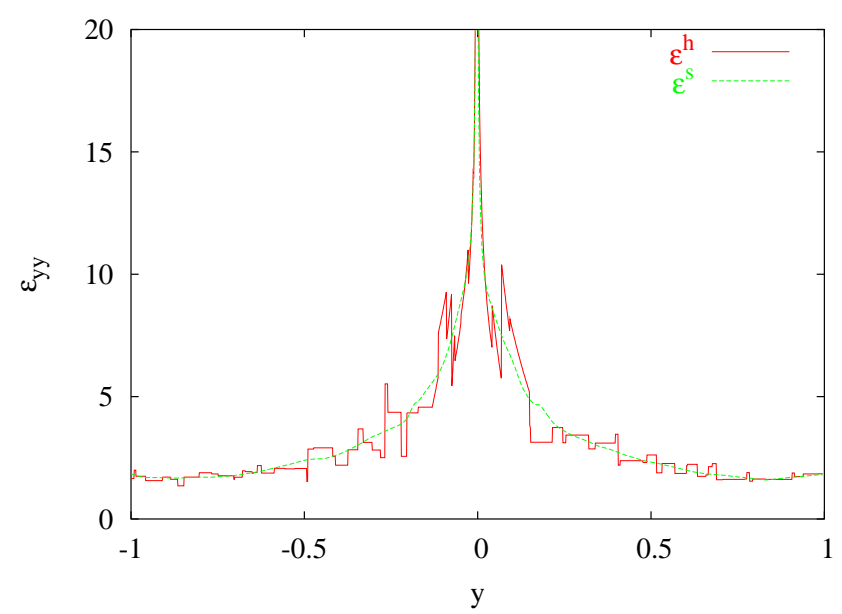

Figure 19: 3D edge crack under mode I loading: plot of $\varepsilon_{y y}$ along line $x=0$. 


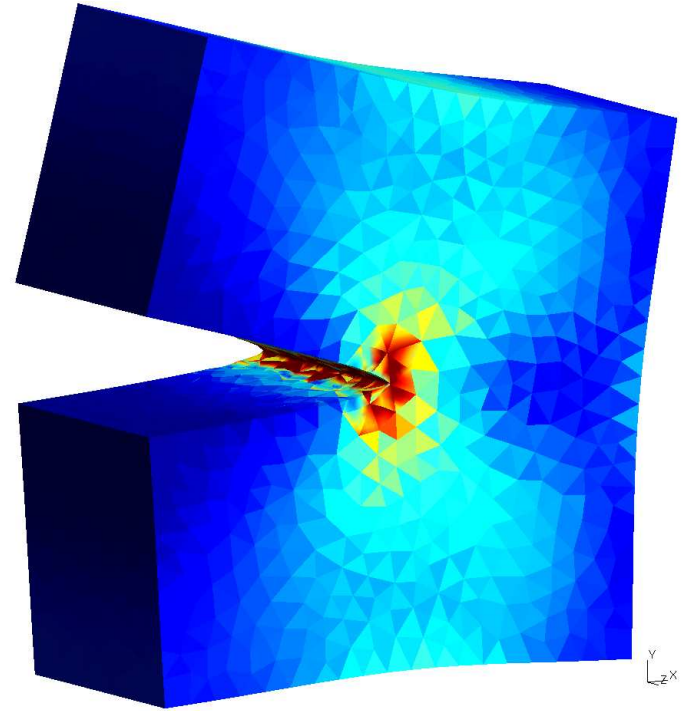

(a) Raw XFEM deformation field

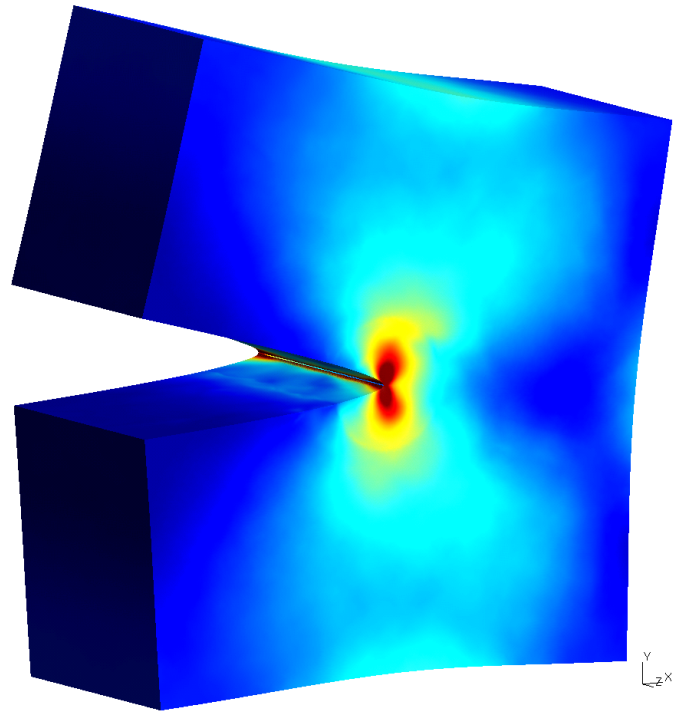

(b) Smoothed (recovered) deformation field

Figure 20: Deformed configuration and strain field for the mode I crack, note that the linear tetrahedra used for the XFEM approximation yield a very rough solution (a) compared to the recovered fields (b).

Additionally, six nodal displacements are fixed so as to avoid rigid body modes. Figure 21 compares the deformation field obtained with the XFEM (a) to the recovered (smoothed) deformation field obtained through MLS derivative recovery. Again, the results are quite satisfying. Figure 22 shows $\varepsilon_{y y}$ along line $x=0$.

\section{Conclusions and extensions}

Through applications to two and three-dimensional fracture, this paper shows how the diffraction method coupled with intrinsically enriched moving least squares approximations provides for a simple and accurate derivative recovery technique for enriched (extended) finite element solutions. In turn, this recovery procedure is used to compute an error estimate whose effectivity index is studied. A numerical convergence study proves that it is necessary to use near-tip enrichment to get optimal convergence rates and effectivity indices that are close to unity. Additionally, the proposed estimator outperforms the SPR-based estimator of Reference [49] for the problems studied.

The next steps to further develop and improve the proposed methodology would be, in the authors' minds, the following:

- Treat more complex cases, with complex geometries and curved cracks, where the use of level sets may be beneficial

- Extend to other enrichment types such as discontinuous derivative enrichments and test the method on multimaterial problems

- Handle the case of multiple tips, which requires defining the polar coordinates adequately for the computation of the near-tip fields associated with both crack tips

- Now that an error estimator is available, it is natural to think about local remeshing. Several remeshing steps could be imagined at each crack propagation step, in order to limit the global error. Additionally, it will be interesting to see the influence of an adapted mesh on crack path. 


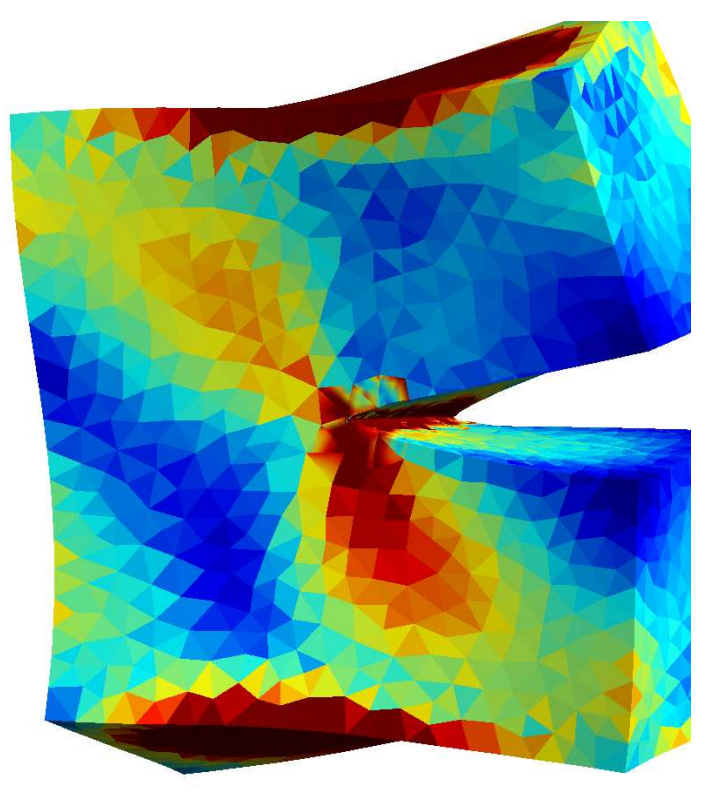

(a) Raw XFEM deformation field

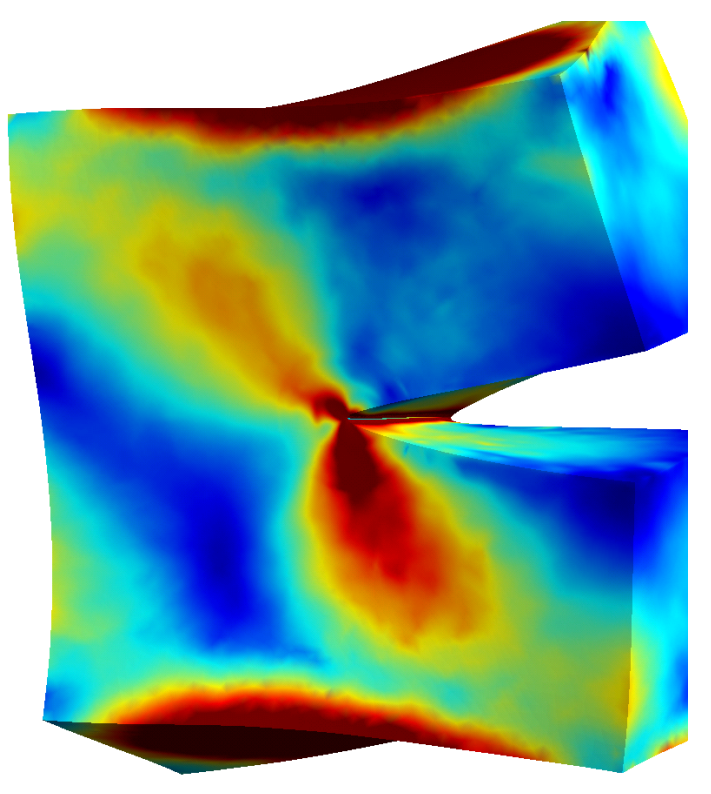

(b) Smoothed (recovered) deformation field

Figure 21: Deformed configuration and strain field for the combined tension/torsion loading case of the 3D crack. Here again, the smoothing fulfills its role nicely.

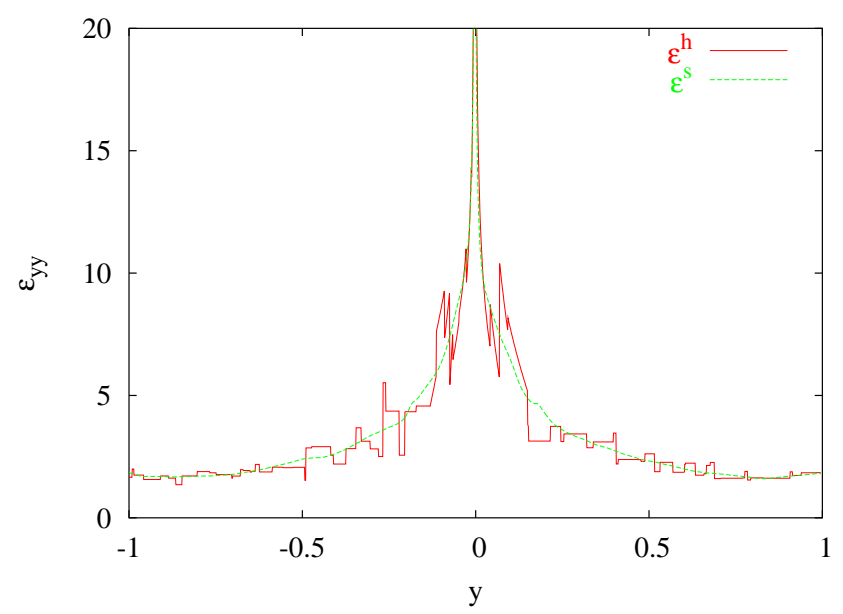

Figure 22: 3D edge crack under mixed mode loading: plot of $\varepsilon_{y y}$ along line $x=0$. 
- In fracture mechanics, the goal of the computation is the obtention of fracture parameters ( $J$ or interaction integrals, stress intensity factors). The authors are developing a posteriori goal-oriented error estimates for the extended finite element method in fracture mechanics

- To improve computational efficiency of the approach, it would be desirable to limit the enriched area (in the MLS approximation) to regions where enrichment is useful, as opposed to using an enriched basis for all points in the domain. The question then translates into the management of the transition area between enriched and non-enriched MLS approximations.

- Another alternative to compute the error estimator would be to use XMLS close to the tip, and SPR elsewhere.

- It could also be beneficial to construct the XMLS shape functions with weight functions $w$ defined using the XFEM mesh as opposed to circles or spheres. This is similar in principle to the work of the intrinsic XFEM [20].

\section{Acknowledgements}

The first author wishes to thank Dr Raphaël Bonnaz for very helpful discussions on error estimates during their common time at EPFL, Lausanne, Switzerland. Mr. Nguyen Vinh Phu is thanked for his dedication and contribution to some of the figures, borrowed from his M.Sc. dissertation. The authors are grateful to Dr Marcus Rüter and Dr Sergey Korotov for their input on goal-oriented error estimation and to Arnaud Fran cois from CENAERO, Belgium, for his help with the SPR comparison. 


\section{References}

[1] M. Ainsworth and A. W. Craig. A posteriori error estimators in the finite element method. Numerische Mathematik, 60:429-463, 1992.

[2] M. Ainsworth and J. T. Oden. A posteriori error estimation in finite element analysis. Computer Methods in Applied Mechanics and Engineering, 142:1-88, 1997.

[3] M. Ainsworth and T. Oden. A unified approach to a posteriori error estimation using element residual methods. Numerische Mathematik, 65(1):23-50, December 1993.

[4] Mark Ainsworth and J. Tinsley Oden. A posteriori error estimation in finite element analysis. Wiley, 2000. ISBN: 0-471-29411-X.

[5] P.M.A. Areias and T. Belytschko. A comment on the article "a finite element method for simulation of strong and weak discontinuities in solid mechanics". Computer Methods in Applied Mechanics and Engineering, 195(912):1275-1276, 2006.

[6] I. Babuška and W. C. Rheinboldt. Error estimates for adaptive finite element computations. SIAM Journal of Numerical Analysis, 15(4):736-754, August 1978.

[7] I. Babuška and I. Melenk. Partition of unity method. International Journal for Numerical Methods in Engineering, 40(4):727-758, 1997.

[8] J. Barlow. Optimal stress locations in finite element models. International Journal for Numerical Methods in Engineering, pages 243-251, 1976.

[9] E. Béchet, H. Minnebo, N. Moës, and B. Burgardt. Improved implementation and robustness study of the $\mathrm{x}$-fem for stress analysis around cracks. International Journal for Numerical Methods in Engineering, 2005.

[10] T. Belytschko and T. Black. Elastic crack growth in finite elements with minimal remeshing. International Journal for Numerical Methods in Engineering, 45:601-620, 1999.

[11] T. Belytschko, Y. Krongauz, M. Fleming, D. Organ, and W. K. Liu. Smoothing and accelerated computations in the element-free Galerkin method. Journal of Computational and Applied Mathematics, 74:111-126, 1996.

[12] T. Belytschko, Y. Y. Lu, and L. Gu. Element-free Galerkin methods. International Journal for Numerical Methods in Engineering, 37:229-256, 1994.

[13] S. Bordas and B. Moran. Enriched finite elements and level sets for damage tolerance assessment of complex structures. Engineering Fracture Mechanics, 73:1176-1201, 2006.

[14] Stéphane Bordas and Antoine Legay. Extended finite element short course. EPFL school of continuing education, Lausanne, Switzerland, December 2005.

[15] J. Chessa and T. Belytschko. The extended finite element method for two-phase fluids. ASME Journal of Applied Mechanics, 70(1):10-17, 2003.

[16] J. Chessa, P. Smolinski, and T. Belytschko. The extended finite element method (x-fem) for solidification problems. International Journal of Numerical Methods in Engineering, 53:1957-1977, 2002.

[17] M. Duflot. A study of the representation of cracks with level sets. International Journal for Numerical Methods in Engineering, to appear, 2006.

[18] M. Duflot and H. Nguyen-Dang. A meshless method with enriched weight functions for fatigue crack growth. International Journal for Numerical Methods in Engineering, 59:1945-1961, 2004.

[19] M. Fleming, Y.A. Chu, B. Moran, and T. Belytschko. Enriched element free Galerkin methods for singular fields. International Journal for Numerical Methods in Engineering, 40:1483-1504, 1997.

[20] T.-P. Fries and T. Belytschko. The intrinsic XFEM: a method for arbitrary discontinuities without additional unknowns. International Journal for Numerical Methods in Engineering, 2006. in press.

[21] A. Gravouil, N. Moës, and T. Belytschko. Non-planar 3d crack growth by the extended finite element and level sets. part II: level set update. International Journal for Numerical Methods in Engineering, 53:2569-2586, 2002.

[22] A. Hansbo and P. Hansbo. A finite element method for the simulation of strong and weak discontinuities in solid mechanics. Computer Methods in Applied Mechanics and Engineering, 193(33-35):3523-3540, 2004. 
[23] P. Laborde, J. Pommier, Y. Renard, and M. Salaun. High order extended finite element method for cracked domains. International Journal for Numerical Methods in Engineering, 190(47):6183 -6200, 2004.

[24] P. Ladevèze and D. Leguillon. Error estimate procedure in the finite element method and applications. SIAM Journal of Numerical Analysis, pages 485-509, 1983.

[25] P. Lancaster and K. Salkauskas. Surfaces generated by moving least squares methods. Mathematics of Computation, 37:141-158, 1981.

[26] A. Legay, J. Chessa, and T. Belytschko. An Eulerian-Lagrangian method for fluid-structure interaction based on level sets. Computer Methods in Applied Mechanics and Engineering, 195(17-18):2070-2087, 2006.

[27] J. M. Melenk and I. Babuška. The partition of unity finite element method: Basic theory and applications. Computer Methods in Applied Mechanics and Engineering, 139:289-314, 1996.

[28] N. Moës and T. Belytschko. Extended finite element method for cohesive crack growth. Engineering Fracture Mechanics, 69:813-834, 2002.

[29] N. Moës, J. Dolbow, and T. Belytschko. A finite element method for crack growth without remeshing. International Journal for Numerical Methods in Engineering, 46:131-150, 1999.

[30] N. Moës, A. Gravouil, and T. Belytschko. Non-planar 3d crack growth by the extended finite element and level sets. part I: Mechanical model. International Journal for Numerical Methods in Engineering, 53:2549-2568, 2002 .

[31] D. Organ, M. Fleming, T. Terry, and T. Belytschko. Continuous meshless approximations for nonconvex bodies by diffraction and transparency. Computational Mechanics, 18:225-235, 1996.

[32] Nguyen Vinh Phu. An object oriented approach to the x-fem with applications to fracture mechanics. Master's thesis, EMMC, November 2005.

[33] T. Rabczuk, S. Bordas, and G. Zi. A three-dimensional meshfree method for continuous crack initiation, nucleation and propagation in statics and dynamics. Computational Mechanics, 2006. in press.

[34] J.-F. Remacle and C. Geuzaine. Gmsh finite element grid generator, 1998. Available at www.geuz.org/gmsh.

[35] B. Moran S. Bordas, R. Duddu and D. Chopp. A combined extended finite element and level set method for biofilm growth. International Journal for Numerical Methods in Engineering, 2006. submitted.

[36] F. Stazi, E. Budyn, J. Chessa, and T. Belytschko. An extended finite element method with higher-order elements for curved cracks. Computational Mechanics.

[37] T. Strouboulis, I. Babuška, and K. Copps. The design and analysis of the generalized finite element method. Computer Methods in Applied Mechanics and Engineering, 181:43-69, 2000.

[38] T. Strouboulis, K. Copps, and I. Babuška. The generalized finite element method : An example of its implementation and illustration of its performance. International Journal for Numerical Methods in Engineering, 47(8):1401-1417, 2000.

[39] T. Strouboulis, L. Zhang, D. Wang, and I. Babuška. A posteriori error estimation for generalized finite element methods. Computer Methods in Applied Mechanics and Engineering, 195(9-12):852-879, February 2006.

[40] N. Sukumar, D. L. Chopp, and B. Moran. Extended finite element method and fast marching method for three-dimensional fatigue crack propagation. Engineering Fracture Mechanics, 70(1):29—48, 2003.

[41] N. Sukumar, D.L. Chopp, N. Moës, and T. Belytschko. Modeling holes and inclusions by level sets in the extended finite element method. International Journal for Numerical Methods in Engineering, 190(47):61836200, 2001.

[42] N. Sukumar, N. Moës, T. Belytschko, and B. Moran. Extended Finite Element Method for three-dimensional crack modelling. International Journal for Numerical Methods in Engineering, 48(11):1549-1570, 2000.

[43] N. Sukumar, B. Moran, T. Black, and T. Belytschko. An element-free galerkin method for three-dimensional fracture mechanics. Computational Mechanics, 20:170-175, 1997.

[44] M. Tabbara, T. Blacker, and T. Belytschko. Finite element derivative recovery by moving least square interpolants. Computer Methods in Applied Mechanics and Engineering, 117:211-223, 1994. 
[45] G. Ventura. On the elimination of quadrature subcells for discontinuous functions in the extended finite element method. International Journal for Numerical Methods in Engineering, 66(5):761-795, 30 April 2006.

[46] G. Ventura, J. X. Xu, and T. Belytschko. Level set crack propagation modelling in the element-free galerkin method. In European Conference on Computational Mechanics, June, 2001.

[47] G. J. Wagner, N. Moës, W.K. Liu, and T. Belytschko. The extended finite element method for Stokes flow past rigid cylinders. International Journal for Numerical Methods in Engineering, 51:393-413, 2001.

[48] Q. Z. Xiao and B. L. Karihaloo. Improving the accuracy of xfem crack tip fields using higher order quadrature and statically admissible stress recovery. International Journal for Numerical Methods in Engineering, 66, 2006.

[49] O. C. Zienkiewicz and J. Z. Zhu. A simple error estimator and adaptive procedure for practical engineering analysis. International Journal for Numerical Methods in Engineering, 24:337-357, 1987.

[50] O. C. Zienkiewicz and J. Z. Zhu. The superconvergent patch recovery and a posteriori error estimates. Part 1: The recovery technique. International Journal for Numerical Methods in Engineering, 33:77:1331-1364, 1992.

[51] O. C. Zienkiewicz and J. Z. Zhu. The superconvergent patch recovery and a posteriori error estimates. Part 2: error estimates and adaptivity. International Journal for Numerical Methods in Engineering, 33:77:1365-1382, 1992.

[52] O.C. Zienkiewicz and Taylor. The finite element method. McGraw Hill, London, 1994. 